\title{
Generalized optical theorems for the reconstruction of Green's function of an inhomogeneous elastic medium
}

\author{
Ludovic Margerin, Dynamique Terrestre et Planétaire, \\ Observatoire Midi-Pyrénées, C.N.R.S., \\ 14 Avenue Edouard Belin, Toulouse, France \\ Haruo Sato, Department of Geophysics, \\ Graduate School of Science, Tohoku University, \\ Aramaki-Aza Aoba 6-3, Aoba-ku, Sendai, Miyagi, 980-8578 JAPAN
}

September 17, 2018

\begin{abstract}
This paper investigates the reconstruction of elastic Green's function from the cross-correlation of waves excited by random noise in the context of scattering theory. Using a general operator equation, -the resolvent formula-, Green's function reconstruction is established when the noise sources satisfy an equipartition condition. In an inhomogeneous medium, the operator formalism leads to generalized forms of optical theorem involving the off-shell $T$-matrix of elastic waves, which describes scattering in the near-field. The role of temporal absorption in the formulation of the theorem is discussed. Previously established symmetry and reciprocity relations involving the on-shell $T$-matrix are recovered in the usual far-field and infinitesimal absorption limits. The theory is applied to a point scattering model for elastic waves. The $T$-matrix of the point scatterer incorporating all recurrent scattering loops is obtained by a regularization procedure. The physical significance of the point scatterer is discussed. In particular this model satisfies the off-shell version of the generalized optical theorem. The link between equipartition and Green's function reconstruction in a scattering medium is discussed.
\end{abstract}

\section{Introduction}

Recently, there has been increasing interest in the use of coda and scattered waves to monitor temporal variations in dynamic structures such as volcanoes and faults. Such an approach is known as codawave interferometry in seismology and diffusive wave spectroscopy in acoustics (Snieder et al., 2002; Snieder and Page, 2007). While the early developments of the technique were based on the use of repeating small earthquakes termed "doublets" (Poupinet et al., 1984, 2008), the possibility to reconstruct Green's function from ambient seismic noise has given rise to a new technique termed passive image interferometry (Sens-Schönfelder and Wegler, 2006; Wegler and Sens-Schoenfelder, 2007; Brenguier et al., 2008a,b). In coda-wave interferometry, temporal changes in the medium are detected by comparing the coda portions of the cross-correlation function of ambient noise, that were computed at different times. Therefore, understanding the basic physical processes that allow the reconstruction of scattered waves from the cross-correlation of random noise sources is of interest in seismology and acoustics.

The reconstruction of Green's function from field cross-correlations has a rich and vast history as reviewed by Shapiro (2011). Green's function retrieval in seismology and elastodynamics finds its roots in the fluctuation-dissipation theorem and in the theory of speckle correlations in optics. In recent years, the pioneering results described by Shapiro (2011) have been extended to different system configurations: open or closed, and to different types of excitations: deterministic or random sources located on a surface 
or distributed in a volume. For instance, general theorems on the reconstruction of Green's function have been established for a variety of inhomogeneous, dissipative (Wapenaar et al., 2006; Snieder et al., 2007), and even non-reciprocal media (Wapenaar, 2006). An important ingredient of seismic interferometry is Green's theorem or its elastodynamic generalization, i.e. the Rayleigh-Betti reciprocity theorem (Ramirez and Weglein, 2009). The importance of Green's theorem and its generalizations for the reconstruction of Green's function is well illustrated by Wapenaar and Fokkema (2006). Depending on the excitation mechanism -active or passive-, the acquisition geometry -surface or borehole measurements-, the nature of boundary conditions -radiation or reflection-, the type of Green's function employed -analytical, numerical, empirical-, a great variety of interferometric methods can be derived from the application of Green's theorem. A comprehensive review of applications to exploration seismology, emphasizing the strengths and limitations of each method is provided by Ramirez and Weglein (2009).

More specifically, the connection between the cross-correlation of wavefields and Green's function between two points in an inhomogeneous elastic half-space has been established by Wapenaar (2004), based on Betti's reciprocity theorem. The reconstruction of Green's function of a homogeneous elastic medium has been discussed by Sánchez-Sesma and Campillo (2006) from a different perspective. These authors find that a set of uncorrelated plane $P$ and $S$ waves verifying the equipartition relation, i.e. carrying the same amount of energy, allow the elastic Green's function to be reconstructed from the crosscorrelation of noise wavefields. This result was extended to the case of one cylindrical inclusion in a 2-D elastic medium by Sánchez-Sesma et al. (2006). These authors demonstrated the complete reconstruction of Green's function including scattered waves in the case of an illumination of the heterogeneous medium by an equipartition mixture of plane $P$ and $S$ waves.

The case of scattering media has been studied by Sato $(2009,2010)$. He demonstrated the reconstruction of singly-scattered coda waves from random noise sources in an acoustic medium composed of point-like velocity perturbations. He considered both volumetric and surface distribution of noise sources. An important connection between energy conservation in scattering and the reconstruction of scattered waves in Green's function obtained from cross-correlations of random noise sources has recently been put forward by Snieder et al. (2008, 2009). Considering a scattering medium illuminated by noise sources distributed on an enclosing surface, Snieder et al. (2008) showed that the reconstruction of Green's function of scalar waves is equivalent to a generalized optical theorem. Such theorems provide general symmetry relations that must be obeyed by the scattering amplitude, which describes the amplitude and phase relations between incident and scattered waves. Lu et al. (2011) extended the method of Snieder et al. (2008) to the case of vector elastic waves, for a scattering medium composed of a homogeneous and isotropic heterogeneity. Snieder and Fleury (2010) and Margerin and Sato (2011) developed a theory for the reconstruction of scattered arrivals in Green's function obtained from the cross-correlation of noise signals in an acoustic medium, for surface and volumetric distributions of noise sources, respectively. These authors demonstrate the cancellation of spurious terms in the multiple scattering series expansion of Green's function cross-correlations by repeated application of the optical theorem.

In this work, we analyze the case of a general -possibly anisotropic- heterogeneity embedded in an isotropic medium, illuminated by a volumetric distribution of random, uncorrelated forces. In the same spirit as Snieder et al. (2008), Lu et al. (2011), and Margerin and Sato (2011), we establish the equivalence between Green's function reconstruction and some symmetry relations pertaining to the scattering of elastic waves which we refer to as "generalized optical theorems". Compared to our previous work on scalar waves, we first use a general operator formalism to establish in a concise way Green's function reconstruction theorem for a general -homogeneous or inhomogeneous- elastic medium. Next, we apply the theorem to Green's function of a scattering medium to establish general symmetry relations in the scattering process. An analysis based on scattering theory finally shows that the symmetry relations are extensions of generalized forms of the optical theorem for elastic waves. The central result of this paper -Equation (61)- contains as special cases all forms of optical theorems found in the literature. In view of future applications to a collection of scatterers we develop a simple point-like scattering model for elastic waves. The formulas (80)-(81) present an extension of the point-scattering model originally developed for scalar waves to the case of vector elastic waves. The physical interpretation of the model is discussed in details. 


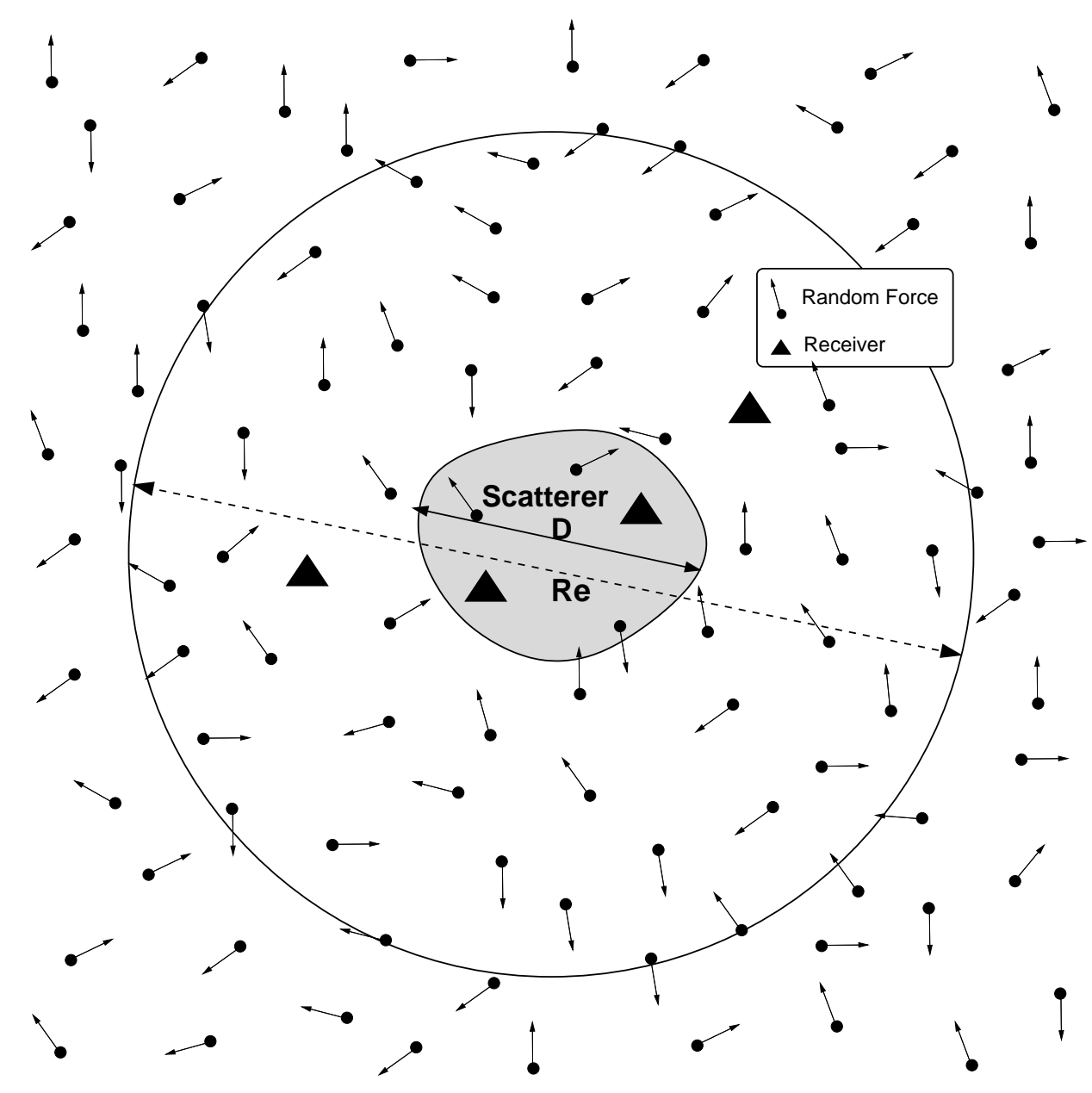

Figure 1: Problem setting. We consider the reconstruction of Green's function including the scattered waves from the cross-correlation of random wavefields recorded in a homogeneous isotropic elastic medium containing an arbitrary heterogeneity of typical dimension $D$ (grey region). Receivers may be placed at arbitrary positions in the medium, including the near-field or the interior of the scatterer. The applied forces are stationary in time, randomly and uniformly oriented, and randomly and homogeneously distributed. The effective radius of the sources is $R_{e}=c_{s} \tau$ with $\tau$ the absorption time and $c_{s}$ the shear wavespeed. The effective radius is (much) larger than the size of the heterogeneity $D$ and the typical distance between the receivers. 
The problem setting is depicted in Figure 1. We consider an arbitrary heterogeneity with bounded support embedded in an elastic medium illuminated by a randomly homogeneous distribution of forces oriented in all possible space directions. By considering the correlation of the displacements recorded at two points $A$ and $B$, we will show the possibility to reconstruct the elastic Green's tensor including the waves scattered by the heterogeneity. In a heterogeneous, slightly dissipative elastic medium, the wavefield generated by random forces $\mathbf{f}(\mathbf{x}, t)$ satisfies the following equation:

$$
\begin{aligned}
\rho_{0}\left(\frac{\partial^{2} u_{i}(\mathbf{x}, t)}{\partial t^{2}}+\frac{1}{\tau} \frac{\partial u_{i}(\mathbf{x}, t)}{\partial t}\right)+\delta \rho(\mathbf{x}) \frac{\partial^{2} u_{i}(\mathbf{x}, t)}{\partial t^{2}}-\partial_{x_{j}} C_{i j k l}^{0} \partial_{x_{k}} u_{l}(\mathbf{x}, t) & \\
& -\partial_{x_{j}} \delta C_{i j k l} \partial_{x_{k}} u_{l}(\mathbf{x}, t)=f_{i}(\mathbf{x}, t)
\end{aligned}
$$

where $\rho_{0}$ and $C_{i j k l}^{0}=\lambda_{0} \delta_{i j} \delta_{k l}+\mu_{0}\left(\delta_{i k} \delta_{j l}+\delta_{i l} \delta_{j k}\right)$ denote the density and elasticity tensor of the isotropic matrix. The summation over repeated indices is assumed. The second term on the left-hand side introduces a characteristic absorption time $\tau$. The deviations of the density and elastic properties from homogeneity are encapsulated in the terms $\delta \rho=\rho-\rho_{0}$ and $\delta C_{i j k l}=C_{i j k l}-C_{i j k l}^{0}$. No particular symmetry properties, except the usual ones, are imposed on $C_{i j k l}$. On the right-hand side of equation (11), we consider a randomly homogeneous and stationary distribution of forces with uniform random orientations. In addition, we assume that the spatial correlation of these forces is much shorter than any other scale length of the problem, which implies that they are well described by a white noise process in space. The basic observable in seismology is the cross-correlation of signals averaged over an ensemble of noise sources:

$$
C_{i j}\left(\mathbf{x}_{B}, \mathbf{x}_{A} ; t\right)=\lim _{T \rightarrow+\infty} \frac{1}{T} \int_{-T / 2}^{T / 2}\left\langle\left\langle u_{i}\left(\mathbf{x}_{B} ; t^{\prime}\right) u_{j}\left(\mathbf{x}_{A} ; t^{\prime}-t\right)^{*}\right\rangle\right\rangle t^{\prime}
$$

where the double brackets $\langle\langle\cdot\rangle\rangle$ denote the ensemble average. If the noise sources are statistically stationary, the basic quantity to be evaluated is the integrand on the right-hand side of equation (2). Note that the definition of the cross-correlation adopted in Eq. (2) differs from that found in other references (see e.g. Wapenaar, 2004). For deterministic or stationary random signals, the change of variable $t^{\prime} \rightarrow t^{\prime}+t$ allows us to recover the form of cross-correlation used in other references. The difference is therefore purely notational. Since calculations are much easier to perform in the frequency domain, we will develop a theory for the cross-spectral density:

$$
C_{i j}\left(\mathbf{x}_{B} ; \mathbf{x}_{A} ; \omega\right)=\int_{-\infty}^{\infty}\left\langle\left\langle u_{i}\left(\mathbf{x}_{B} ; t^{\prime}\right) u_{j}\left(\mathbf{x}_{A} ; t^{\prime}-t\right)^{*}\right\rangle\right\rangle e^{i \omega t} d t
$$

In equation (3), the left-hand side does not depend on the variable $t^{\prime}$ for a stationary signal. Upon introducing the spectral representation of the random process $u_{i}(\mathbf{x}, t)$ :

$$
u_{i}(\mathbf{x} ; t)=\frac{1}{2 \pi} \int_{-\infty}^{+\infty} u_{i}(\mathbf{x} ; \omega) e^{-i \omega t} d \omega
$$

we obtain the following basic relation:

$$
\left\langle\left\langle u_{i}\left(\mathbf{x}_{B} ; \omega^{\prime}\right) u_{j}\left(\mathbf{x}_{A} ; \omega\right)^{*}\right\rangle\right\rangle=2 \pi C_{i j}\left(\mathbf{x}_{B}, \mathbf{x}_{A} ; \omega\right) \delta\left(\omega-\omega^{\prime}\right) .
$$

The delta function condition which appears in equation (44) is characteristic of random processes that are stationary in time. We note that the usual Fourier transforms $u_{i}(\mathbf{x} ; \omega)$ may not be well defined since we are interested in stationary random fields. A physically satisfying solution is to define the Fourier transforms for a finite observation time $T$, and to take the limit $T \rightarrow \infty$ after averaging over noise sources. Instead, we make use of the probabilistic interpretation of the spectral representation (4), where the coefficients of the exponential is itself a random process, and the equality is to be understood in the probabilistic (mean-squared) sense (Rytov et al., 1989; Yaglom, 2004). Since only the cross-spectral density of the field is needed in this work, and this quantity can be defined by traditional Fourier analysis, the formal 
representation (4) suffices. A link between the physical and mathematical approaches is provided by the periodogram:

$$
C_{i j}\left(\mathbf{x}_{B}, \mathbf{x}_{A} ; \omega\right)=\lim _{T \rightarrow \infty} \frac{1}{T}\left\langle\left\langle u_{i}^{T}\left(\mathbf{x}_{B} ; \omega\right) u_{j}^{T}\left(\mathbf{x}_{A} ; \omega\right)^{*}\right\rangle\right\rangle,
$$

where $u_{i}^{T}(\mathbf{x} ; \omega)$ represents the Fourier transform of the original signal observed over a finite time window $T$. As is well-known, Equation (6) expresses the fact that the periodogram is an asymptotically unbiased estimator of the cross-spectral density.

The paper is organized as follows. In section 2, we introduce some important notations and present a simple derivation of Green's function reconstruction for a homogeneous medium illuminated by a set of randomly oriented and spatially uncorrelated forces. In section 3 we introduce the Dirac calculus which will be used to generalize our results to an inhomogeneous medium. The formalism is applied to demonstrate the reconstruction of Green's function of a general -homogeneous or inhomogeneouselastic medium illuminated by a homogeneous distribution of randomly oriented forces, or by a wavefield at equipartition. In section 4 , we introduce the $T$-operator for elastic waves and discuss its symmetry properties. In section 5, we explore the reconstruction of Green's function for an arbitrary inhomogeneity embedded in a homogeneous medium and establish several forms of the generalized optical theorem for elastic waves. In section 6, we establish the generalized optical theorem from the governing equations of scattering in the limit of infinitesimal absorption. In section 7 we consider a simple configuration composed of a single point scatterer and demonstrate the Green's function reconstruction including all the recurrent scattering loops. In section 8 our results are discussed in the framework of equipartition theory and compared to other works.

\section{Green's function reconstruction in a homogeneous medium}

In this section, we introduce important notations and present in a simplified context our approach to Green's function reconstruction. Although it is not the main theme of the paper, we give a short derivation of Green's function reconstruction for elastic waves excited by random uncorrelated noise sources in a homogeneous medium. Our assumptions differ from the one adopted by Sánchez-Sesma and Campillo (2006) who considered a set of uncorrelated plane $P$ and $S$ waves at equipartition. The complete equivalence between the two models of noise source will be demonstrated later in the paper. Let us first rewrite the correlation function (3) in terms of Green's function. The elastic wavefields recorded at two points $\mathbf{x}_{A}$ and $\mathbf{x}_{B}$ due to a distribution of forces can be expressed as:

$$
\begin{aligned}
& u_{i}\left(\mathbf{x}_{B} ; \omega^{\prime}\right)=-\iiint_{-\infty}^{\infty} G_{i k}^{0}\left(\mathbf{x}_{B}, \mathbf{x}_{0} ; \omega^{\prime}\right) f_{k}\left(\mathbf{x}_{0} ; \omega^{\prime}\right) d^{3} x_{0}, \\
& u_{j}\left(\mathbf{x}_{A} ; \omega\right)^{*}=-\iiint_{-\infty}^{\infty} G_{j l}^{0}\left(\mathbf{x}_{A}, \mathbf{x}_{1} ; \omega\right)^{*} f_{l}\left(\mathbf{x}_{1} ; \omega\right)^{*} d^{3} x_{1},
\end{aligned}
$$

where $G^{0}(\omega)$ is Green's function of a homogeneous, slightly dissipative elastic medium at angular frequency $\omega$. Using the summation convention over repeated indices, $G^{0}(\omega)$ is recognized as the fundamental solution to the equation of elastodynamics:

$$
\left(\mu_{0} \partial x_{l} \partial x_{l} \delta_{i j}+\left(\lambda_{0}+\mu_{0}\right) \partial_{x_{i}} \partial_{x_{j}}\right) G_{j k}^{0}\left(\boldsymbol{x}, \boldsymbol{x}^{\prime} ; \omega\right)+\rho_{0}(\omega+i / 2 \tau)^{2} G_{i k}^{0}\left(\boldsymbol{x}, \boldsymbol{x}^{\prime} ; \omega\right)=\delta_{i k} \delta\left(\boldsymbol{x}, \boldsymbol{x}^{\prime}\right)
$$

and has the following analytical form:

$$
G_{i k}^{0}\left(\mathbf{x}, \mathbf{x}^{\prime} ; \omega\right)=\left(g^{s}(\mathbf{r} ; \omega)+g^{n}(\mathbf{r} ; \omega)\right)\left(\delta_{i k}-\hat{r}_{i} \hat{r}_{k}\right)+\left(g^{p}(\mathbf{r} ; \omega)-2 g^{n}(\mathbf{r} ; \omega)\right) \hat{r}_{i} \hat{r}_{k},
$$

where Green's function has been split into transverse and longitudinal parts. In equation (9) we have introduced the notation $\mathbf{r}=\mathbf{x}-\mathbf{x}^{\prime}$, the unit vector $\hat{\mathbf{r}}$ in the direction of $\mathbf{r}$, and the functions $g^{p}, g^{s}$ and 
$g^{n}:$

$$
\begin{aligned}
g^{s}(\mathbf{r} ; \omega) & =-\frac{e^{i k_{s} r}}{4 \pi r \rho_{0} c_{s}^{2}} \\
g^{p}(\mathbf{r} ; \omega) & =-\frac{e^{i k_{p} r}}{4 \pi r \rho_{0} c_{p}^{2}} \\
g^{n}(\mathbf{r} ; \omega) & =\frac{1}{4 \pi r^{2} \rho_{0}}\left(\frac{i k_{p} e^{i k_{p} r}-i k_{s} e^{i k_{s} r}}{\omega_{+}^{2}}+\frac{1}{r \omega_{+}^{2}}\left(e^{i k_{s} r}-e^{i k_{p} r}\right)\right) .
\end{aligned}
$$

The following short-hand notations have been used: $\omega^{+}=\omega+i / 2 \tau, k_{p}=\omega_{+} / c_{p}$ and $k_{s}=\omega_{+} / c_{s}$, where $c_{p}$ and $c_{s}$ are the $P$ and $S$ wave velocities in the background medium with Lamé parameters $\lambda_{0}$ and $\mu_{0}$. The definition of Green's function adopted in equation (9) obeys an opposite sign convention to that usually found in the seismological literature. This choice will be clarified in section 3, In equation (9), we have explicitly separated the far-field terms $g^{s}$ and $g^{p}$ from the near field term $g^{n}$.

Equation (77) allows us to express the cross-correlation of wavefields measured at $\mathbf{x}_{A}$ and $\mathbf{x}_{B}$ in the frequency domain as follows:

$$
\begin{aligned}
& \left\langle\left\langle u_{i}\left(\mathbf{x}_{B} ; \omega^{\prime}\right) u_{j}\left(\mathbf{x}_{A} ; \omega\right)^{*}\right\rangle\right\rangle= \\
& \iiint_{\mathbb{R}^{6}} G_{i k}^{0}\left(\mathbf{x}_{B}, \mathbf{x}_{0} ; \omega^{\prime}\right) G_{j k}^{0}\left(\mathbf{x}_{A}, \mathbf{x}_{1} ; \omega\right)^{*}\left\langle\left\langle f_{k}\left(\mathbf{x}_{0} ; \omega^{\prime}\right) f_{l}\left(\mathbf{x}_{1} ; \omega\right)^{*}\right\rangle\right\rangle d^{3} x_{0} d^{3} x_{1}
\end{aligned}
$$

For spatially uncorrelated and randomly oriented forces, one further assumes that

$$
\left\langle\left\langle f_{k}\left(\mathbf{x}_{0} ; \omega^{\prime}\right) f_{l}\left(\mathbf{x}_{1} ; \omega\right)^{*}\right\rangle\right\rangle=2 \pi \delta_{k l} \delta\left(\mathbf{x}_{0}-\mathbf{x}_{1}\right) S(\omega) \delta\left(\omega-\omega^{\prime}\right),
$$

where $S(\omega)$ is the power spectral density of the forces. Therefore the key integral to be computed writes

$$
C_{i j}\left(\mathbf{x}_{B}, \mathbf{x}_{A} ; \omega\right)=S(\omega) \iiint_{-\infty}^{\infty} G_{i k}^{0}\left(\mathbf{x}_{B}, \mathbf{x} ; \omega\right) G_{j k}^{0}\left(\mathbf{x}_{A}, \mathbf{x} ; \omega\right)^{*} d^{3} x .
$$

In coordinate space, Green's function has the complicated form (9). However, using Fourier transforms, it is found that Green's function has the following exact expression in wavenumber space:

$$
G_{i j}^{0}\left(\mathbf{k}^{\prime}, \mathbf{k} ; \omega\right)=g^{l}(\mathbf{k} ; \omega) \delta\left(\mathbf{k}^{\prime}-\mathbf{k}\right) \hat{k}_{i} \hat{k}_{j}+g^{t}(\mathbf{k} ; \omega) \delta\left(\mathbf{k}^{\prime}-\mathbf{k}\right)\left(\delta_{i j}-\hat{k}_{i} \hat{k}_{j}\right),
$$

where we have introduced the following notations:

$$
g^{l}(\mathbf{k} ; \omega)=\frac{1}{\rho_{0}\left(\omega^{2}-c_{p}^{2} k^{2}+i \omega / \tau\right)}, \quad \quad g^{t}(\mathbf{k} ; \omega)=\frac{1}{\rho_{0}\left(\omega^{2}-c_{s}^{2} k^{2}+i \omega / \tau\right)},
$$

and the unit vector $\hat{\mathbf{k}}$ is in the direction of $\mathbf{k}$. In equation (14), Green's function has been separated into longitudinal and transverse parts. Upon inverse Fourier transformation, both contribute to the near-field term in the spatial domain. In wavenumber space, the key integral is defined as

$$
\tilde{C}_{i j}\left(\mathbf{k}^{\prime}, \mathbf{k} ; \omega\right)=\frac{S(\omega)}{(2 \pi)^{3}} \iiint_{\mathbb{R}^{6}} C_{i j}\left(\mathbf{x}_{B}, \mathbf{x}_{A} ; \omega\right) e^{-i \mathbf{k}^{\prime} \cdot \mathbf{x}_{B}+i \mathbf{k} \cdot \mathbf{x}_{A}} d^{3} x_{A} d^{3} x_{B}
$$

Inserting the spectral representation of Green's function in equation (13), one obtains the following key integral in the wavenumber domain:

$$
\tilde{C}_{i j}\left(\mathbf{k}^{\prime}, \mathbf{k} ; \omega\right)=S(\omega) \iiint_{-\infty}^{+\infty} G_{i k}^{0}\left(\mathbf{k}^{\prime}, \mathbf{k}_{0} ; \omega\right) G_{j k}^{0}\left(\mathbf{k}, \mathbf{k}_{0} ; \omega\right)^{*} d^{3} k_{0}
$$


Reporting expression (14) into equation (17), one obtains:

$$
\tilde{C}_{i j}\left(\mathbf{k}^{\prime}, \mathbf{k} ; \omega\right)=-\frac{S(\omega) \tau}{\rho_{0} \omega} \operatorname{Im} g^{l}(\mathbf{k}) \delta\left(\mathbf{k}^{\prime}-\mathbf{k}\right) \hat{k}_{i} \hat{k}_{j}-\frac{S(\omega) \tau}{\rho_{0} \omega} \operatorname{Im} g^{t}(\mathbf{k}) \delta\left(\mathbf{k}^{\prime}-\mathbf{k}\right)\left(\delta_{i j}-\hat{k}_{i} \hat{k}_{j}\right)
$$

which establishes the reconstruction of Green's function of a homogeneous, slightly dissipative elastic medium. More precisely, equations (3), (11) and (18) allow us to obtain the following closed form expression of the cross-correlation function of two wavefields in the space-time domain:

$$
\begin{aligned}
\frac{d}{d t} \lim _{T \rightarrow \infty} \frac{1}{T} \int_{-T / 2}^{T / 2}\left\langle\left\langle u_{i}\left(\boldsymbol{x}_{B}, t^{\prime}\right) u_{j}\left(\boldsymbol{x}_{A}, t^{\prime}-t\right)\right\rangle\right\rangle d t^{\prime}= \\
\frac{\tau}{2 \rho_{0}(2 \pi)} \int_{-\infty}^{\infty} d \omega\left[e^{-i \omega t} G_{i j}^{0}\left(\boldsymbol{x}_{B}, \boldsymbol{x}_{A}, \omega\right)-e^{-i \omega(-t)} G_{i j}^{0}\left(\boldsymbol{x}_{B}, \boldsymbol{x}_{A}, \omega\right)\right] S(\omega) .
\end{aligned}
$$

Hence, we retrieve the classical result that the temporal derivative of the cross-correlation of two wavefields is proportional to the difference of the retarded and advanced Green's functions filtered by the source power spectral density. Strictly speaking, the limit and integral signs on the left-hand side of equation (19) are superfluous. However, introducing the temporal averaging process makes a closer link with the measurement procedure. It is worth noting that the result (19) is valid under the assumption of equal absorption times for $P$ and $S$ waves. We will come back to this point in section 8 ,

\section{Green's function reconstruction in an inhomogeneous medium}

\subsection{Formalism and notations}

To perform the calculations required for the proof of the elastic Green's function reconstruction in an inhomogeneous medium, it is convenient to introduce the Dirac formalism. This approach allows us to switch easily from coordinate to wavenumber space and is extremely efficient to perform compact formal derivations. In this paper, we work in a linear space of vector wavefunctions in which various representations can be introduced. It is convenient to think of this vector space as the tensor product of a 3-dimensional polarization space, and the usual space of scalar wavefunctions. In what follows, the symbol $\langle\mathbf{u} \mid \mathbf{v}\rangle$ will be employed to denote the scalar product between two vectors. For instance, the space of vector wavefunctions is equipped with the following scalar product:

$$
\langle\mathbf{u} \mid \mathbf{v}\rangle=\iiint_{-\infty}^{+\infty} u_{i}(\mathbf{x})^{*} v_{i}(\mathbf{x}) d^{3} x
$$

which is inherited from the tensor product structure. In what follows, we will switch among various representations by introducing generalized orthonormal bases which verify the continuum normalization. The three most important bases for this work are listed below.

(1) First, we define: $|i, \mathbf{x}\rangle=\left|\hat{\mathbf{e}}_{i}\right\rangle \otimes|\mathbf{x}\rangle$, where the symbol $\otimes$ denotes a tensor or direct product between vectors of the polarization and wavefunction space, respectively. The bra-ket notations and definitions adopted here follow the treatment of vector fields in quantum mechanics (see Messiah, 1999, p.549-551). The vectors $\left|\hat{\mathbf{e}}_{i}\right\rangle$ form an orthonormal basis of the 3-D polarization space. The simplest choice is to take $\left|\hat{\mathbf{e}}_{i}\right\rangle$ as unit vectors along the axes of a right-handed orthogonal reference frame. We adopt the convention that unit vectors are denoted by a hat. The symbol $|\mathbf{x}\rangle$ denotes generalized eigenvectors of the position operator. By forming the tensor product $\left|\hat{\mathbf{e}}_{i}\right\rangle \otimes|\mathbf{x}\rangle$, one obtains a wavefunction which is perfectly localized in space at position $\mathbf{x}$ and perfectly polarized along direction $\hat{\mathbf{e}}_{i}$. The completeness of this set of orthonormal vectors is intuitively clear and the following normalization of the basis vectors is easily verified: $\left\langle\mathbf{x}, i \mid j, \mathbf{x}^{\prime}\right\rangle=\left\langle\hat{\mathbf{e}}_{i} \mid \hat{\mathbf{e}}_{j}\right\rangle\left\langle\mathbf{x} \mid \mathbf{x}^{\prime}\right\rangle=\delta_{i j} \delta\left(\mathbf{x}-\mathbf{x}^{\prime}\right)$. Using this basis, we recover the usual definition of the recorded elastic wavefield in cartesian coordinates

$$
|\mathbf{u}\rangle=\sum_{i} \iiint_{-\infty}^{+\infty} u_{i}(\mathbf{x})|i, \mathbf{x}\rangle d^{3} x .
$$




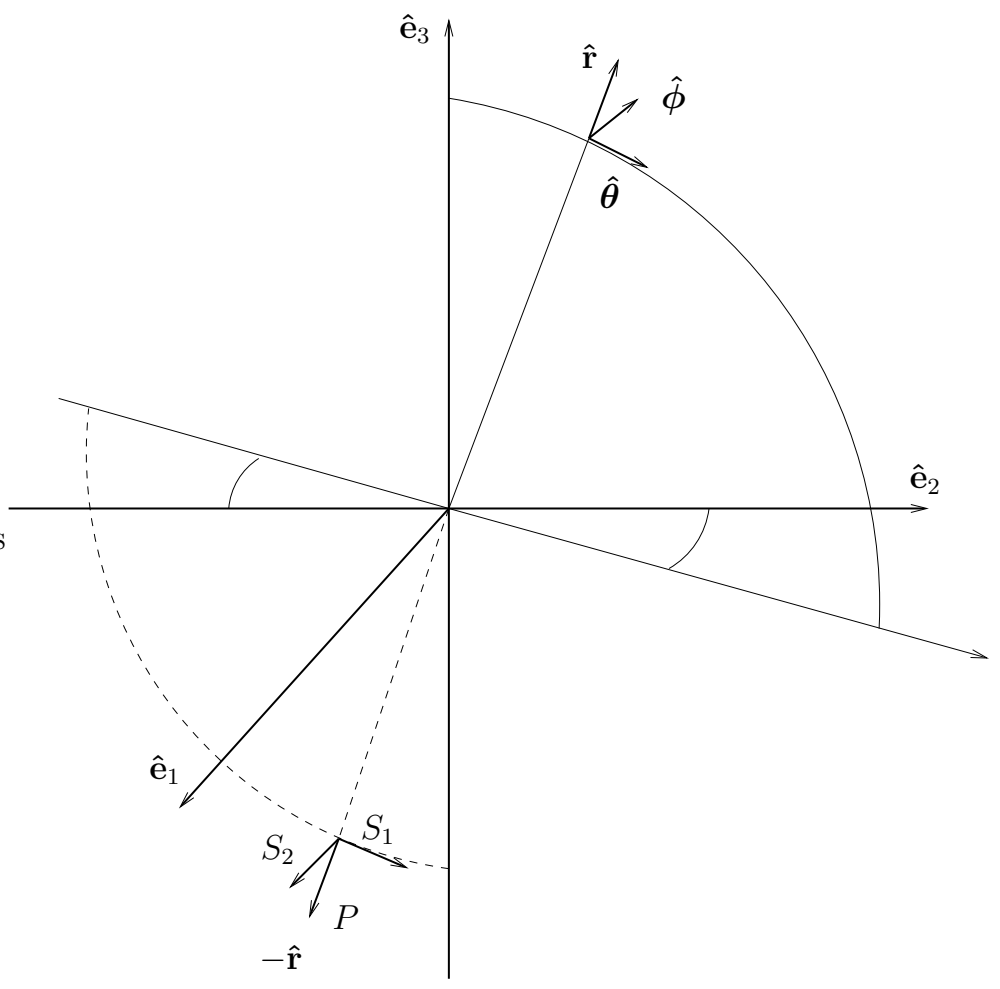

Figure 2: Conventions adopted for the definition of the elastic wave polarizations $\left\{S_{1}, S_{2}, P\right\}$. ( $\left.\hat{\mathbf{e}}_{1}, \hat{\mathbf{e}}_{2}, \hat{\mathbf{e}}_{3}\right)$ denotes a right-handed cartesian system, to which the local right-handed spherical system $(\hat{\boldsymbol{\theta}}, \hat{\boldsymbol{\phi}}, \hat{\mathbf{r}})$ is referred to. An inversion of the propagation direction $\hat{\mathbf{r}} \rightarrow-\hat{\mathbf{r}}$, implies $(\hat{\boldsymbol{\theta}}, \hat{\boldsymbol{\phi}}) \rightarrow(\hat{\boldsymbol{\theta}},-\hat{\boldsymbol{\phi}})$. The three polarizations $S_{1}, S_{2}$ and $P$ are shown for the propagation direction $-\hat{\mathbf{r}}$. 
(2) Next, we introduce the symbol $|i, \mathbf{k}\rangle=\left|\hat{\mathbf{e}}_{i}\right\rangle \otimes|\mathbf{k}\rangle$ which denote a basis of eigenvectors of the vector Laplace operator with eigenvalue $-\mathbf{k}^{2}$. The symbol $|\mathbf{k}\rangle$ represents a plane wave with wavenumber $\mathbf{k}$ which obeys the normalization of the continuum. By forming the tensor product $\left|\hat{\mathbf{e}}_{i}\right\rangle \otimes|\mathbf{k}\rangle$, one obtains a plane wave perfectly polarized along direction $\left|\hat{\mathbf{e}}_{i}\right\rangle$ and propagating in direction $\hat{\mathbf{k}}$. In the coordinate representation (1) introduced above, the eigenfunctions $|i, \mathbf{k}\rangle$ write

$$
\langle\mathbf{x}, i \mid j, \mathbf{k}\rangle=\frac{\delta_{i j} e^{i \mathbf{k} \cdot \mathbf{x}}}{(2 \pi)^{3 / 2}}
$$

(3) Finally, we denote by $|\alpha, \mathbf{k}\rangle$ the eigenvectors of the elastodynamic operator in free space $L^{0}$, which is defined as

$$
\left\langle\mathbf{x}, i\left|L^{0}\right| \mathbf{u}\right\rangle=-\left(\lambda_{0}+\mu_{0}\right) \partial_{x_{i}} \partial_{x_{j}} u_{j}(\boldsymbol{x})-\mu_{0} \partial x_{j} \partial x_{j} u_{i}(\boldsymbol{x}) .
$$

The notational conventions adopted in Eq. (23) are as follows: the operator $L^{0}$ acts on the vector wavefunction $|\mathbf{u}\rangle$ from the left to give a new wavefunction $|\mathbf{v}\rangle=L^{0}|\mathbf{u}\rangle$, and the right-hand side gives the usual expression of the wavefunction $|\mathbf{v}\rangle$ in coordinate space. The minus sign adopted in definition (23) makes $L^{0}$ a positive operator, i.e., such that $\left\langle u\left|L^{0}\right| u\right\rangle \geq 0$. This property is easily proved since $\left\langle u\left|L^{0}\right| u\right\rangle / 4$ represents the deformation energy of the solid (see Ben-Menahem and Singh (1998) p.31, for details). In coordinate space, the eigenvectors of $L^{0}$ are expressed as:

$$
\langle\mathbf{x}, i \mid \alpha, \mathbf{k}\rangle=\frac{\hat{p}_{i}^{\alpha} e^{i \mathbf{k} \cdot \mathbf{x}}}{(2 \pi)^{3 / 2}}
$$

where $\hat{\mathbf{p}}^{\alpha}$ denotes the polarization vector and $\mathbf{k}$ is the wavevector. Equation (24) introduces the complete orthonormal set of linearly polarized plane $\mathrm{P}$ and $\mathrm{S}$ waves. In what follows, latin subscripts refer to cartesian coordinates (representations 1 and 2), while greek letters will be used to distinguish the contribution of the 3 different polarizations $\left\{S_{1}, S_{2}, P\right\}$ in representation 3 . The $\hat{\mathbf{p}}^{\alpha}$ form a right-handed basis in polarization space which coincides with the usual spherical coordinate frame $(\hat{\boldsymbol{\theta}}, \hat{\boldsymbol{\phi}}, \hat{\mathbf{r}}=\hat{\mathbf{k}})$, where $\hat{\boldsymbol{\theta}}$ and $\hat{\boldsymbol{\phi}}$ point in the direction of increasing $\theta$ and $\phi$, respectively. Our conventions for the definition of the polarizations $\left\{S_{1}, S_{2}, P\right\}$ are depicted in Figure 2, It is important to keep in mind that polarization vectors rotate with the incident wavevector $\mathbf{k}$. Note that with our notational conventions, there should be no risk of confusion between representations (2) and (3).

\subsection{Resolvent formula and Green's function retrieval}

We now generalize the previous derivation to the case of an inhomogeneous elastic medium. We will consider simultaneously the case of a wavefield at equipartition, or a system driven by a white noise distribution of random forces. Our formalism allows us to treat the two cases on the same footing. Some basic facts about resolvents and their connection with Green's functions are recalled hereafter. Let us first introduce the operator $L$ which pertains to elastic waves in inhomogeneous media:

$$
L=L^{0}+V\left(\omega_{0}^{2}\right)
$$

where $L^{0}$ is the elastodynamic operator in free space introduced in equation (23) and $V\left(\omega_{0}^{2}\right)$ is the scattering potential at the frequency $\omega_{0}$ :

$$
\left\langle\mathbf{x}, i\left|V\left(\omega_{0}^{2}\right)\right| l, \mathbf{x}^{\prime}\right\rangle=-\delta \rho(\mathbf{x}) \omega_{0}^{2} \delta\left(\mathbf{x}-\mathbf{x}^{\prime}\right) \delta_{i l}-\partial_{x_{j}} \delta C_{i j k l}(\mathbf{x}) \partial_{x_{k}} \delta\left(\mathbf{x}-\mathbf{x}^{\prime}\right),
$$

where the usual summation convention over repeated index is used. Equation (26) defines the matrix elements of the scattering potential operator in the coordinate representation. This representation is most usually encountered in the Green's function retrieval literature. For simplicity, we assume that the scattering potential vanishes outside a bounded region of $\mathbb{R}^{3}$. To demonstrate that $V\left(\omega_{0}^{2}\right)$ is Hermitian, we let $V\left(\omega_{0}^{2}\right)$ act on test functions $\mathbf{f}$ and $\mathbf{g}$. Since the Hermitian symmetry of the term involving the 
density perturbation $\delta \rho$ is easily verified, we only consider the $\delta C_{i j k l}$ term:

$$
\begin{aligned}
\left\langle\mathbf{f}\left|V\left(\omega_{0}^{2}\right)\right| \mathbf{g}\right\rangle & =\iiint_{\mathbb{R}^{3}} f_{i}(\mathbf{x})^{*} \partial_{x_{j}}\left(\delta C_{i j k l}(\mathbf{x}) \partial_{x_{k}} g_{l}(\mathbf{x})\right) d^{3} x \\
& =-\iiint_{\mathbb{R}^{3}}\left(\delta C_{i j k l}(\mathbf{x}) \partial_{x_{j}} f_{i}(\mathbf{x})\right)^{*} \partial_{x_{k}} g_{l}(\mathbf{x}) d^{3} x \\
& =\iiint_{\mathbb{R}^{3}}\left(\partial_{x_{k}}\left(\delta C_{i j k l}(\mathbf{x}) \partial_{x_{j}} f_{i}(\mathbf{x})\right)^{*} g_{l}(\mathbf{x}) d^{3} x\right. \\
& =\iiint_{\mathbb{R}^{3}}\left(\partial_{x_{k}}\left(\delta C_{k l i j}(\mathbf{x}) \partial_{x_{j}} f_{i}(\mathbf{x})\right)\right)^{*} g_{l}(\mathbf{x}) d^{3} x=\left\langle\mathbf{g}\left|V\left(\omega_{0}^{2}\right)\right| \mathbf{f}\right\rangle^{*} .
\end{aligned}
$$

In the derivation of equation (27), we have used integration by parts twice and the usual symmetries of the elasticity tensor: $C_{i j k l}=C_{k l i j}=C_{j i k l}=C_{i j l k}$. In the following, we assume that all Hermitian operators we have to deal with have unique self-adjoint extensions. Because the elastic tensor and density fields are real, relation (27) implies that the matrix elements of the scattering potential in the representation (1) obey the following symmetry relation:

$$
\left\langle\mathbf{x}, i\left|V\left(\omega_{0}^{2}\right)\right| j, \mathbf{x}^{\prime}\right\rangle=V_{i j}\left(\mathbf{x}, \mathbf{x}^{\prime} ; \omega_{0}^{2}\right)=V_{j i}\left(\mathbf{x}^{\prime}, \mathbf{x} ; \omega_{0}^{2}\right)
$$

Let us introduce the resolvent of a linear operator $L$ as follows:

$$
(\lambda \mathcal{I}-L) R(\lambda)=\mathcal{I}
$$

where $\mathcal{I}$ is the identity operator in the space of vector wavefunctions, and $\operatorname{Im} \lambda \neq 0$. Comparison of equations (29) and (9) reveals that $G^{0}(\omega)$ is intimately related to $R^{0}(\lambda)$, the resolvent of $L^{0}$. By making the following substitutions $\lambda \rightarrow \rho_{0}(\omega+i / 2 \tau)^{2}, L \rightarrow L^{0}, R \rightarrow G^{0}(\omega)$ in equation (29), we can formally define Green's function at angular frequency $\omega$ with a small, finite absorption time $\tau$. This justifies a posteriori the definition of Green's function adopted in section 2. With this choice, $G^{0}(\omega)$ is related to the resolvent of a positive operator with continuous spectrum on the positive real axis (see Equation (23) and the discussion that follows). Let us now introduce in a similar fashion the resolvent and Green's function of the equation of elastodynamics in an inhomogeneous medium as follows:

$$
\begin{gathered}
{\left[\lambda \mathcal{I}-\left(L^{0}+V\left(\omega_{0}^{2}\right)\right)\right] R(\lambda)=\mathcal{I},} \\
{\left[\rho_{0}(\omega+i / 2 \tau)^{2} \mathcal{I}-\left(L^{0}+V\left(\omega_{0}^{2}\right)\right)\right] G(\omega)=\mathcal{I} .}
\end{gathered}
$$

An important point to be noted in the definition (31) is the fact that $\omega$, the real part of the complex frequency, does not necessarily equal $\omega_{0}$, the angular frequency at which the scattering potential is evaluated. For elastic waves, the only physically accessible Green's function is obtained when we set $\omega=\omega_{0}$ in equation (31). Taking the matrix elements of equation (31) in representation (1), we recover the fact that $G_{i m}\left(\mathbf{x}, \mathbf{x}^{\prime} ; \omega_{0}\right)$ is the solution of Equation (1) with the force density $f_{i}=-\delta_{i m} \delta\left(\mathbf{x}-\mathbf{x}^{\prime}\right)$, as it should. In equation (31), everything happens as if the scattering potential has been "frozen" at the frequency $\omega_{0}$. This mathematical trick allows us to use the standard tools of potential scattering theory and facilitates intermediate calculations. In elastodynamics, this idea has previously been introduced by Budreck and Rose (1991). Eventually, all key formulas will be obtained for $\omega=\omega_{0}$ and will involve physically accessible quantities only.

Let us consider the elastic wavefield at frequency $\omega_{1}$ excited by a distribution of forces and recorded at $\mathbf{x}_{B}$ in an inhomogeneous medium:

$$
\begin{aligned}
& u_{i}\left(\mathbf{x}_{B} ; \omega_{1}\right)=-\sum_{k} \iiint_{-\infty}^{\infty}\left\langle\mathbf{x}_{B}, i\left|G\left(\omega_{1}\right)\right| k, \mathbf{x}_{1}\right\rangle\left\langle k, \mathbf{x}_{1} \mid f\right\rangle d^{3} x_{1} \\
& u_{i}\left(\mathbf{x}_{B} ; \omega_{1}\right)=-\sum_{\beta} \iiint_{-\infty}^{\infty}\left\langle\mathbf{x}_{B}, i\left|G\left(\omega_{1}\right)\right| \beta, \mathbf{k}_{1}\right\rangle\left\langle\beta, \mathbf{k}_{1} \mid f\right\rangle d^{3} k_{1},
\end{aligned}
$$


In equation (32a), we have used the coordinate representation to express the field generated by the force distribution. But any other complete set of vectors can be inserted between $G$ and $f$. As an example, in equation (32b), we make use of this freedom by introducing a decomposition of the identity operator using the complete orthonormal set (3), which results in a hybrid representation. Indeed, the symbol $\left\langle\mathbf{x}_{B}, i\left|G\left(\omega_{1}\right)\right| \beta, \mathbf{k}_{1}\right\rangle=G_{i \beta}\left(\mathbf{x}_{B}, \mathbf{k}_{1} ; \omega_{1}\right)$ has its left foot in representation (1) and its right foot in representation (3). The quantity $G_{i \beta}\left(\mathbf{x}_{B}, \mathbf{k}_{1} ; \omega_{1}\right)$ can be calculated from the usual coordinate representation of Green's function $G_{i j}\left(\mathbf{x}_{B}, \mathbf{x}_{1}\right)$ by Fourier transformation over the variable $\mathbf{x}_{1}$ and by expanding the standard basis $\left|\hat{\mathbf{e}}_{j}\right\rangle$ over the rotating polarization basis $\left|\hat{\mathbf{p}}^{\beta}\right\rangle$. Clearly, the quantity $\tilde{f}_{\beta}\left(\mathbf{k}_{1}\right)=$ $\left\langle\beta, \mathbf{k}_{1} \mid f\right\rangle$ gives the amplitude of the plane wave with polarization $\beta$ and wavenumber $\mathbf{k}_{1}$, which is excited by the force distribution. As previously noted, if the force distribution is statistically homogeneous, the wavenumber decomposition of the field has a formal character. Standard Fourier analysis can nevertheless be used after ensemble averaging (Rytov et al. $(1989))$. Using equations (32a) $-(32 \mathrm{~b})$, the cross-correlation between the wavefields at $\mathbf{x}_{A}$ and $\mathbf{x}_{B}$ can be written as:

$$
\begin{aligned}
& \left\langle\left\langle u_{i}\left(\mathbf{x}_{B} ; \omega_{1}\right) u_{j}\left(\mathbf{x}_{A} ; \omega_{0}\right)^{*}\right\rangle\right\rangle= \\
& \sum_{k, l} \iiint_{\mathbb{R}^{6}}\left\langle\mathbf{x}_{B}, i\left|G\left(\omega_{1}\right)\right| k, \mathbf{x}_{1}\right\rangle\left\langle\mathbf{x}_{0}, l\left|G\left(\omega_{0}\right)^{\dagger}\right| j, \mathbf{x}_{A}\right\rangle\left(\left\langle f_{k}\left(\mathbf{x}_{1} ; \omega_{1}\right) f_{l}\left(\mathbf{x}_{0} ; \omega_{0}\right)^{*}\right\rangle\right) d^{3} x_{0} d^{3} x_{1} \\
& \left\langle\left\langle u_{i}\left(\mathbf{x}_{B} ; \omega_{1}\right) u_{j}\left(\mathbf{x}_{A} ; \omega_{0}\right)^{*}\right\rangle\right\rangle= \\
& \sum_{\alpha, \beta} \iiint_{\mathbb{R}^{6}}\left\langle\mathbf{x}_{B}, i\left|G\left(\omega_{1}\right)\right| \beta, \mathbf{k}_{1}\right\rangle\left\langle\mathbf{k}_{0}, \alpha\left|G\left(\omega_{0}\right)^{\dagger}\right| j, \mathbf{x}_{A}\right\rangle\left(\left\langle\tilde{f}_{\beta}\left(\mathbf{k}_{1} ; \omega_{1}\right) \tilde{f}_{\alpha}\left(\mathbf{k}_{0} ; \omega_{0}\right)^{*}\right\rangle\right) d^{3} k_{0} d^{3} k_{1},
\end{aligned}
$$

where $G\left(\omega_{0}\right)^{\dagger}$ denotes the Hermitian adjoint of $G\left(\omega_{0}\right)$. We now consider two possible physical assumptions regarding the excitations that drive the system. As in equation (12), we may assume that the forces are randomly oriented and spatially delta-correlated. Alternatively, we may consider that the medium is illuminated by a set of plane $P$ and $S$ waves whose amplitudes constitute a white-noise in modal space:

$$
\left\langle\left\langle\tilde{f}_{\beta}\left(\mathbf{k}_{1} ; \omega_{1}\right) \tilde{f}_{\alpha}\left(\mathbf{k}_{0} ; \omega_{0}\right)^{*}\right\rangle\right\rangle=2 \pi \delta_{\alpha \beta} \delta\left(\mathbf{k}_{0}-\mathbf{k}_{1}\right) S\left(\omega_{0}\right) \delta\left(\omega_{0}-\omega_{1}\right)
$$

Assumption (34) may be interpreted as a requirement of equipartition: the system is illuminated by uncorrelated plane $P$ and $S$ waves coming from all possible directions with equal weights. This point will be further substantiated in section 8 . Under which conditions such an equipartition state can be achieved in practice remains to be elucidated. Reporting Equations (12) and (34) in Equations (33a) and (33b) respectively, we find:

$$
\begin{aligned}
C_{i j}\left(\mathbf{x}_{B} ; \mathbf{x}_{A} ; \omega_{0}\right) & =S\left(\omega_{0}\right) \sum_{k} \iiint_{-\infty}^{\infty}\left\langle\mathbf{x}_{B}, i\left|G\left(\omega_{0}\right)\right| k, \mathbf{x}\right\rangle\left\langle k, \mathbf{x}\left|G\left(\omega_{0}\right)^{\dagger}\right| j, \mathbf{x}_{A}\right\rangle d^{3} x \\
C_{i j}\left(\mathbf{x}_{B} ; \mathbf{x}_{A} ; \omega_{0}\right) & =S\left(\omega_{0}\right) \sum_{\alpha} \iiint_{-\infty}^{\infty}\left\langle\mathbf{x}_{B}, i\left|G\left(\omega_{0}\right)\right| \alpha, \mathbf{k}_{0}\right\rangle\left\langle\mathbf{k}_{0}, \alpha\left|G\left(\omega_{0}\right)^{\dagger}\right| j, \mathbf{x}_{A}\right\rangle d^{3} k_{0} \\
& =S\left(\omega_{0}\right)\left\langle\mathbf{x}_{B}, i\left|G\left(\omega_{0}\right) G\left(\omega_{0}\right)^{\dagger}\right| j, \mathbf{x}_{A}\right\rangle,
\end{aligned}
$$

where in the last equation, we have used the completeness of the bases (1) and (3), respectively. In both cases -white noise distribution of forces, or wavefield at equipartition- we have reduced the computation of the cross-correlation of two random wavefields to the evaluation of the matrix elements of the operator $G\left(\omega_{0}\right) G\left(\omega_{0}\right)^{\dagger}$. In fact, from the defining property of Green's function as the resolvent of a self-adjoint operator -equation (31)-, the following identity follows:

$$
\begin{aligned}
G\left(\omega_{0}\right) G\left(\omega_{0}\right)^{\dagger} & =R\left(\rho_{0}\left(\omega_{0}+i / 2 \tau\right)^{2}\right) R\left(\rho_{0}\left(\omega_{0}-i / 2 \tau\right)^{2}\right) \\
& =-\frac{i \tau}{\rho_{0} \omega_{0}}\left(R\left(\rho_{0}\left(\omega_{0}+i / 2 \tau\right)^{2}\right)-R\left(\rho_{0}\left(\omega_{0}-i / 2 \tau\right)^{2}\right)\right) \\
& =-\frac{\tau}{2 i \rho_{0} \omega_{0}}\left(G\left(\omega_{0}\right)-G\left(\omega_{0}\right)^{\dagger}\right),
\end{aligned}
$$


with $R(\lambda)$ the resolvent operator introduced in Equation (30). The key point in the above derivation is the use of a fundamental operator equation known as the "first resolvent formula" in the second line of Equation (36). The resolvent formula is valid for a broad class of operators (closed linear operators) including the differential operators considered in this work (Richtmyer, 1978). The last step follows simply from the Hermitian character of the elastodynamic operator $L^{0}+V\left(\omega_{0}^{2}\right)$. Taking into account the power spectral density of the source and calculating the matrix elements of the right-hand side of equation (36) yields:

$$
\begin{aligned}
\frac{d}{d t} \lim _{T \rightarrow \infty} \frac{1}{T} \int_{-T / 2}^{T / 2}\left\langle\left\langle u_{i}\left(\boldsymbol{x}_{B}, t^{\prime}\right) u_{j}\left(\boldsymbol{x}_{A}, t^{\prime}-t\right)\right\rangle\right\rangle d t^{\prime}= \\
\frac{\tau}{2 \rho_{0}(2 \pi)} \int_{-\infty}^{\infty} d \omega\left[e^{-i \omega t} G_{i j}\left(\boldsymbol{x}_{B}, \boldsymbol{x}_{A}, \omega\right)-e^{-i \omega(-t)} G_{j i}\left(\boldsymbol{x}_{A}, \boldsymbol{x}_{B}, \omega\right)\right] S(\omega),
\end{aligned}
$$

where the Hermitian symmetry of $G(\omega)$ has been used. When the reciprocity theorem applies, the integrand in the right-hand side of equation (37) is recognized as the usual imaginary part of Green's function. Thus, the derivative of the cross-correlation tensor of random signals yields a filtered version of the difference between advanced and retarded Green's functions. Since $G$ can be substituted with the Green's function of any self-adjoint operator, this implies the reconstruction of Green's function for other systems governed by linear wave equations. Derivations of Green's function reconstruction based on a different operator formalism have been further developped by Wapenaar et al. (2006) and Snieder et al. (2007). Our derivation highlights the fact that a wavefield at equipartition and a random distribution of white noise sources are indistinguishable from the point of view of cross-correlation functions. The complete equivalence between the two types of excitations will be demonstrated in section 8 . The general result (36) does not constitute, however, the end of our investigations. We will show that interesting information on the scattering of elastic waves can be obtained by expressing the Green's function of an inhomogeneous medium using the formalism of scattering theory. To do so, basic notions of scattering of elastic waves are recalled in the next section.

\section{Summary of scattering of elastic waves}

Our starting point is the observation that Equation (31) is formally identical to a Schrödinger equation, with a scattering potential $V\left(\omega_{0}^{2}\right)$. This allows us to use all the arsenal of scattering theory. In particular we can introduce the retarded Lippman-Schwinger eigenvectors of the operator $\left(L^{0}+V\left(\omega_{0}^{2}\right)\right)$ which satisfy the following equation in the infinitesimal absorption limit $\tau \rightarrow \infty$ (Economou (2006)):

$$
\left|\psi^{\alpha}(k \hat{\mathbf{k}})\right\rangle=|\alpha, k \hat{\mathbf{k}}\rangle+G^{0}\left(c_{\alpha} k\right) V\left(\omega_{0}^{2}\right)\left|\psi^{\alpha}(k \hat{\mathbf{k}})\right\rangle,
$$

with $k>0$. It is worth noting that in equation (38), the potential is "frozen" at the frequency $\omega_{0}$, and is scanned by plane $P$ and $S$ waves with all possible wavevectors $k \hat{\mathbf{k}}$ with $k>0$, and eigenfrequency $\omega=c_{\alpha} k>0$. These eigenvectors do not depend on the sign of $\omega_{0}$. For elastic waves, the physical wavefunction is obtained when the condition $\omega=\omega_{0}=c_{\alpha} k$ is satisfied. For later developments it is nevertheless convenient to introduce the more general Lippmann-Schwinger eigenvectors (38). All the scattering properties of an inhomogeneous elastic medium are conveniently encapsulated in an operator denoted by $T^{\left(\omega_{0}^{2}\right)}$ which is defined as follows:

$$
T^{\left(\omega_{0}^{2}\right)}(\omega)=V\left(\omega_{0}^{2}\right)+V\left(\omega_{0}^{2}\right) G(\omega) V\left(\omega_{0}^{2}\right) .
$$

In equation (39), we have carefully distinguished between the angular frequency of the target $-\omega_{0^{-}}$and of Green's function - $\omega$-. Clearly, the physical result is obtained when $\omega$ equals $\omega_{0}$ and we introduce a special notation for this case:

$$
T^{0}=T^{\left(\omega_{0}^{2}\right)}\left(\omega_{0}\right)
$$


For simplicity, we have dropped the frequency dependence in this definition. The superscript ${ }^{0}$ reminds us of the fact that $\omega=\omega_{0}$. The definition of the $T^{\left(\omega_{0}^{2}\right)}$-operator adopted in equation (39) is in keeping with our definition of Green's function of an inhomogeneous medium (see equation (31)) and will facilitate intermediate calculations in section 6 Using the $T^{\left(\omega_{0}^{2}\right)}$-operator, we can rewrite the Lippman-Schwinger equation as follows $(\tau \rightarrow \infty, k>0)$ :

$$
\left|\psi^{\alpha}(k \hat{\mathbf{k}})\right\rangle=|\alpha, k \hat{\mathbf{k}}\rangle+G^{0}\left(c_{\alpha} k\right) T^{\left(\omega_{0}^{2}\right)}\left(c_{\alpha} k\right)|\alpha, k \hat{\mathbf{k}}\rangle,
$$

which further implies:

$$
V\left(\omega_{0}^{2}\right)\left|\psi^{\alpha}(k \hat{\mathbf{k}})\right\rangle=T^{\left(\omega_{0}^{2}\right)}\left(c_{\alpha} k\right)|\alpha, k \hat{\mathbf{k}}\rangle
$$

Green's function of the heterogeneous medium can also conveniently be rewritten in terms of the $T^{\left(\omega_{0}^{2}\right)}$ operator and the free space Green's function alone:

$$
G(\omega)=G^{0}(\omega)+G^{0}(\omega) T^{\left(\omega_{0}^{2}\right)}(\omega) G^{0}(\omega) .
$$

Since our approach is based on an analogy with quantum scattering, a word of comment about the sign of $\omega_{0}$ is in order at this point. The problem of negative frequencies is easily solved because we consider real fields in the space-time domain. This imposes the following Hermitian symmetry on the matrix elements of $G$ and $T$ in coordinate representation:

$$
G_{i j}\left(\mathbf{x}, \mathbf{x}^{\prime} ;-\omega_{0}\right)=G_{i j}\left(\mathbf{x}, \mathbf{x}^{\prime} ; \omega_{0}\right)^{*} \quad T_{i j}^{\left(\omega_{0}^{2}\right)}\left(\mathbf{x}, \mathbf{x}^{\prime} ;-\omega_{0}\right)=T_{i j}^{\left(\omega_{0}^{2}\right)}\left(\mathbf{x}, \mathbf{x}^{\prime} ; \omega_{0}\right)^{*}
$$

As a consequence, all results obtained for positive frequency are readily translated to negative frequency using the symmetry conditions (44).

In the far-field of the scatterer, i.e. in the limit $x \gg D$ with $\mathbf{x}$ the observation point and $D$ the dimension of the scatterer (Figure 1), we may approximate the free space Green's function as follows:

$$
G_{i j}^{0}\left(\mathbf{x}, \mathbf{x}^{\prime}\right) \approx-\frac{e^{i k_{s} x-i k_{s} \hat{\mathbf{x}} \cdot \mathbf{x}^{\prime}}}{4 \pi \rho_{0} c_{s}^{2} x}\left(\delta_{i j}-\hat{x}_{i} \hat{x}_{j}\right)-\frac{e^{i k_{p} x-i k_{p} \hat{\mathbf{x}} \cdot \mathbf{x}^{\prime}}}{4 \pi \rho_{0} c_{p}^{2} x} \hat{x}_{i} \hat{x}_{j}
$$

where $\mathbf{x}^{\prime}$ denotes an arbitrary point of the scatterer. The position vectors are measured from an arbitrary origin located inside the scattering region and $\hat{\mathbf{x}}$ is a unit vector in the direction of $\mathbf{x}$. The asymptotic formula (45) allows us to express the Lippman-Schwinger wavefunction in the usual coordinate representation as follows:

$$
\left\langle i, \mathbf{x} \mid \psi^{\beta}\left(k^{\beta} \hat{\mathbf{k}}_{b}\right)\right\rangle=\frac{1}{(2 \pi)^{3 / 2}}\left(\hat{q}_{i}^{\beta} e^{i k^{\beta} \hat{\mathbf{k}}_{b} \cdot \mathbf{x}}+\frac{f^{\alpha \leftarrow \beta}\left(\hat{\mathbf{k}}_{a}, \hat{\mathbf{k}}_{b}\right)}{x} \hat{p}_{i}^{\alpha} e^{i k^{\alpha} x}\right),
$$

valid in the limit $x \rightarrow \infty$, where $x=|\mathbf{x}|$. In equation (46), we have introduced the scattering amplitudes:

$$
\begin{aligned}
f^{\alpha \leftarrow \beta}\left(\hat{\mathbf{k}}_{a}, \hat{\mathbf{k}}_{b}\right) & =-\frac{(2 \pi)^{3}}{4 \pi \rho_{0}\left(c_{\alpha}\right)^{2}}\left\langle\alpha, k^{\alpha} \hat{\mathbf{k}}_{a}\left|T^{\left(\omega_{0}^{2}\right)}\left(\omega_{0}\right)\right| \beta, k^{\beta} \hat{\mathbf{k}}_{b}\right\rangle \\
& =-\frac{(2 \pi)^{3}}{4 \pi \rho_{0}\left(c_{\alpha}\right)^{2}} T_{\alpha \beta}^{0}\left(k^{\alpha} \hat{\mathbf{k}}_{a}, k^{\beta} \hat{\mathbf{k}}_{b}\right)
\end{aligned}
$$

and the notations: $k^{\alpha, \beta}=\omega_{0} / c_{\alpha, \beta}>0$. In the last line of equation (47), we have introduced "on-shell" matrix elements of the $T^{0}$-operator. In general, the matrix elements $\left\langle\alpha, \mathbf{k}_{a}\left|T^{\left(\omega_{0}^{2}\right)}(\omega)\right| \beta, \mathbf{k}_{b}\right\rangle$ are said to be "on-shell" when the condition $\omega^{2}=c_{\alpha}^{2} k_{a}^{2}=c_{\beta}^{2} k_{b}^{2}$ is satisfied. Although this property does not depend on the fact that we are on the shell $\omega=\omega_{0}$, we eventually specialize our results to this particular case. The scattering amplitudes contain information on the scattering pattern for all possible mode conversions. For instance, taking $\beta=1, \alpha=3$, corresponds to the physical situation where a linearly polarized incident shear wave is scattered into a compressional wave. Note that the definition is valid for an arbitrary 
anisotropic inhomogeneity. From the symmetries of the scattering potential: $V_{i j}\left(\mathbf{x}, \mathbf{x}^{\prime} ; \omega_{0}^{2}\right)=V_{j i}\left(\mathbf{x}^{\prime}, \mathbf{x} ; \omega_{0}^{2}\right)$ and the reciprocity of the free space Green's function $G_{i j}^{0}\left(\mathbf{x}, \mathbf{x}^{\prime} ; \omega\right)=G_{j i}^{0}\left(\mathbf{x}^{\prime}, \mathbf{x} ; \omega\right)$, we deduce a similar reciprocity relation for the $T^{\left(\omega_{0}^{2}\right)}$ operator:

$$
T_{i j}^{\left(\omega_{0}^{2}\right)}\left(\mathbf{x}, \mathbf{x}^{\prime} ; \omega\right)=T_{j i}^{\left(\omega_{0}^{2}\right)}\left(\mathbf{x}^{\prime}, \mathbf{x} ; \omega\right) .
$$

Using equation (48), our formalism allows us to recover well-known reciprocity relations for the scattering amplitudes:

$$
\begin{aligned}
T_{\alpha \beta}^{\left(\omega_{0}^{2}\right)}\left(\mathbf{k}_{a}, \mathbf{k}_{b} ; \omega\right) & =\sum_{i, j} \frac{1}{(2 \pi)^{3}} \iiint_{\mathbb{R}^{6}} \hat{p}_{i}^{\alpha} e^{-i \mathbf{k}_{a} \cdot \mathbf{x}} T_{i j}^{\left(\omega_{0}^{2}\right)}\left(\mathbf{x}, \mathbf{x}^{\prime} ; \omega\right) \hat{q}_{j}^{\beta} e^{i \mathbf{k}_{b} \cdot \mathbf{x}^{\prime}} d^{3} x d^{3} x^{\prime} \\
& =\sum_{i, j} \frac{1}{(2 \pi)^{3}} \iiint_{\mathbb{R}^{6}} \hat{q}_{j}^{\beta} e^{i \mathbf{k}_{b} \cdot \mathbf{x}^{\prime}} T_{j i}^{\left(\omega_{0}^{2}\right)}\left(\mathbf{x}^{\prime}, \mathbf{x} ; \omega\right) \hat{p}_{i}^{\alpha} e^{-i \mathbf{k}_{a} \cdot \mathbf{x}} d^{3} x d^{3} x^{\prime} \\
& =\operatorname{sgn}(\alpha, \beta) T_{\beta \alpha}^{\left(\omega_{0}^{2}\right)}\left(-\mathbf{k}_{b},-\mathbf{k}_{a} ; \omega\right),
\end{aligned}
$$

where $\operatorname{sgn}(\alpha, \beta)=-1$ for $(\alpha, \beta) \in\{(1,2),(2,1),(1,3),(3,1)\}$ and +1 otherwise. In equation (49), the matrix elements are generally off-shell because the condition $\omega^{2}=c_{\alpha}^{2} k_{a}^{2}=c_{\beta}^{2} k_{b}^{2}$ needs not be satisfied. This relation is in fact far more general than what is required for our purposes. We will therefore specialize it to the on-shell case with the condition $\omega=\omega_{0}$. Note that the sign change $-\operatorname{sgn}(\alpha, \beta)$ - is a mere consequence of the convention adopted to define the polarization vectors and illustrated in Figure 2 Indeed, upon a change of the wavevector $\mathbf{k}_{a} \rightarrow-\mathbf{k}_{a}$, the polarization vectors transform as follows: $\left(\hat{\mathbf{p}}^{1}, \hat{\mathbf{p}}^{2}, \hat{\mathbf{p}}^{3}\right) \rightarrow\left(\hat{\mathbf{p}}^{1},-\hat{\mathbf{p}}^{2},-\hat{\mathbf{p}}^{3}\right)$. As an example, let us consider an incident $S$ wave with polarization vector $\hat{\mathbf{q}}^{1}$ and a scattered $P$ wave with polarization vector $\hat{\mathbf{p}}^{3}$. Application of the symmetry relation (49) to equation (47) yields the following reciprocity relation between the scattering amplitudes:

$$
c_{p}^{2} f^{P \leftarrow S_{1}}\left(\hat{\mathbf{k}}_{a}, \hat{\mathbf{k}}_{b}\right)=-c_{s}^{2} f^{S_{1} \leftarrow P}\left(-\hat{\mathbf{k}}_{b},-\hat{\mathbf{k}}_{a}\right),
$$

a relation which has been noted by a number of authors in the past (Varatharajulu, 1977; Dassios et al., 1987; Aki, 1992). Interestingly, this reciprocity relation shows up without any effort from the formalism we have introduced. Some physical implications of relation (50) will be discussed in section 8 , in connection with the role of equipartition in Green's function retrieval. With the help of our scattering formalism, we will explore the consequences of Green's function reconstruction in a scattering medium in the next section.

\section{Green's function reconstruction in a scattering medium}

As noted in section (2), Green's function reconstruction from homogeneous random sources is a consequence of a basic operator identity. We now explore the consequences of this result in the case of a scattering medium. The identity satisfied by Green's function of the heterogeneous medium writes:

$$
G\left(\omega_{0}\right) G\left(\omega_{0}\right)^{\dagger}=-\frac{\tau}{2 i \rho_{0} \omega_{0}}\left(G\left(\omega_{0}\right)-G\left(\omega_{0}\right)^{\dagger}\right) .
$$

Using equations (43) we can express the left-hand side (L.H.S.) of equation (51) as follows:

$$
\begin{aligned}
G\left(\omega_{0}\right) G\left(\omega_{0}\right)^{\dagger}= & G^{0}\left(\omega_{0}\right) G^{0}\left(\omega_{0}\right)^{\dagger}+G^{0}\left(\omega_{0}\right) G^{0}\left(\omega_{0}\right)^{\dagger} T^{0 \dagger} G^{0}\left(\omega_{0}\right)^{\dagger} \\
& +G^{0}\left(\omega_{0}\right) T^{0} G^{0}\left(\omega_{0}\right) G^{0}\left(\omega_{0}\right)^{\dagger} \\
& +G^{0}\left(\omega_{0}\right) T^{0} G^{0}\left(\omega_{0}\right) G^{0}\left(\omega_{0}\right)^{\dagger} T^{0 \dagger} G^{0}\left(\omega_{0}\right)^{\dagger} .
\end{aligned}
$$

Similarly, we obtain for the right-hand side (R.H.S) of equation (51):

$$
G\left(\omega_{0}\right)-G\left(\omega_{0}\right)^{\dagger}=G^{0}\left(\omega_{0}\right)-G^{0}\left(\omega_{0}\right)^{\dagger}+G^{0}\left(\omega_{0}\right) T^{0} G^{0}\left(\omega_{0}\right)-G^{0}\left(\omega_{0}\right)^{\dagger} T^{0 \dagger} G^{0}\left(\omega_{0}\right)^{\dagger} .
$$


Upon inserting relation (36) into equation (54), Green's function reconstruction formula (51) yields the following identity for the $T$ operator:

$$
\frac{1}{2 i}\left(T^{0}-T^{0 \dagger}\right)=-\frac{\rho_{0} \omega_{0}}{\tau} T^{0} G^{0}\left(\omega_{0}\right) G^{0}\left(\omega_{0}\right)^{\dagger} T^{0 \dagger}
$$

In order to elucidate the physical meaning of equation (56), let us take matrix elements in the representation (3). We find:

$$
\frac{1}{2 i}\left\langle\alpha, \mathbf{k}_{a}\left|\left(T^{0}-T^{0 \dagger}\right)\right| \beta, \mathbf{k}_{b}\right\rangle=\frac{1}{2 i}\left(T_{\alpha \beta}^{0}\left(\mathbf{k}_{a}, \mathbf{k}_{b}\right)-T_{\beta \alpha}^{0}\left(\mathbf{k}_{b}, \mathbf{k}_{a}\right)^{*}\right),
$$

on the L.H.S., and:

$$
\begin{aligned}
-\frac{\rho_{0} \omega_{0}}{\tau}\left\langle\alpha, \mathbf{k}_{a}\left|T^{0} G^{0}\left(\omega_{0}\right) G^{0}\left(\omega_{0}\right)^{\dagger} T^{0 \dagger}\right| \beta, \mathbf{k}_{b}\right\rangle= & \\
& -\frac{\omega_{0}}{\rho_{0} \tau} \sum_{\gamma} \iiint_{\mathbb{R}^{3}} \frac{\left\langle\alpha, \mathbf{k}_{a}\left|T^{0}\right| \gamma, \mathbf{k}\right\rangle\left\langle\gamma, \mathbf{k}\left|T^{0 \dagger}\right| \beta, \mathbf{k}_{b}\right\rangle}{\left(\omega_{0}^{2}-c_{\gamma}^{2} k^{2}\right)^{2}+\omega_{0}^{2} / \tau^{2}} d^{3} k,
\end{aligned}
$$

on the R.H.S., where we have made use of the spectral decomposition of $G^{0}\left(\omega_{0}\right)$, valid irrespective of the sign of $\omega_{0}$ :

$$
G^{0}\left(\omega_{0}\right)=\sum_{\gamma} \iiint_{\mathbb{R}^{3}} \frac{|\gamma, \mathbf{k}\rangle\langle\gamma, \mathbf{k}|}{\rho_{0}\left(\omega_{0}^{2}-c_{\gamma}^{2} k^{2}+i \omega_{0} / \tau\right)} d^{3} k
$$

On the right-hand side of equation (59), the symbol $|\gamma, \mathbf{k}\rangle\langle\gamma, \mathbf{k}|$ is an operator which projects the wavefunctions on the subspace of plane $P$ and $S$ waves with wavevector $\mathbf{k}$. We will now turn equation (58) into a more physically appealing form. First, we note that the representation of the product of Green's function $G^{0}\left(\omega_{0}\right) G^{0}\left(\omega_{0}\right)^{\dagger}$ is left unchanged by the substitution $\mathbf{k} \rightarrow-\mathbf{k}$. Next, we make use of the reciprocity relation (49) to rearrange the numerator as follows:

$$
\left\langle\alpha, \mathbf{k}_{a}\left|T^{0}\right| \gamma,-\mathbf{k}\right\rangle\left\langle\gamma,-\mathbf{k}\left|T^{0 \dagger}\right| \beta, \mathbf{k}_{b}\right\rangle=\operatorname{sgn}(\alpha, \beta)\left\langle\beta,-\mathbf{k}_{b}\left|T^{0 \dagger}\right| \gamma, \mathbf{k}\right\rangle\left\langle\gamma, \mathbf{k}\left|T^{0}\right| \alpha,-\mathbf{k}_{a}\right\rangle,
$$

where we have used $\operatorname{sgn}(\alpha, \gamma) \operatorname{sgn}(\gamma, \beta)=\operatorname{sgn}(\alpha, \beta)$. The following substitutions: $\mathbf{k}_{a} \rightarrow-\mathbf{k}_{b}, \mathbf{k}_{b} \rightarrow-\mathbf{k}_{a}$, $\alpha \rightarrow \beta, \beta \rightarrow \alpha$, leaves equation (57) unchanged except for an additional factor $\operatorname{sgn}(\alpha, \beta)$ which cancels the same factor on the right-hand side of equation (60). We therefore arrive at the following general identity:

$$
\frac{1}{2 i}\left(T_{\alpha \beta}^{0}\left(\mathbf{k}_{a}, \mathbf{k}_{b}\right)-T_{\beta \alpha}^{0}\left(\mathbf{k}_{b}, \mathbf{k}_{a}\right)^{*}\right)=-\frac{\omega_{0}}{\rho_{0} \tau} \sum_{\gamma} \iiint \frac{T_{\gamma \beta}^{0}\left(\mathbf{k}, \mathbf{k}_{b}\right) T_{\gamma \alpha}^{0}\left(\mathbf{k}, \mathbf{k}_{a}\right)^{*}}{\left(\omega_{0}^{2}-c_{\gamma}^{2} k^{2}\right)^{2}+\omega_{0}^{2} / \tau^{2}} d^{3} k,
$$

which we will call the generalized optical theorem in the presence of small finite absorption. Equation (61) is a general identity which must be obeyed by the "off-shell" matrix elements of the $T^{0}$-operator for a general scatterer in an elastic medium. The matrix elements are said to be off-shell because $\mathbf{k}$, $\mathbf{k}_{a}$ and $\mathbf{k}_{b}$ do not have to verify the shell condition $\omega_{0}^{2}=c_{\gamma}^{2} k^{2}$. The physical meaning of these off-shell contributions will be discussed below. Equation (61) is a central result of this paper, from which all forms of optical theorem derived in the literature can be deduced. The justification of the term "generalized optical theorem" is made clear when we consider the limit of infinitesimal absorption $\tau \rightarrow \infty$. Using standard results from distribution theory, we find:

$$
\lim _{\tau \rightarrow \infty}-\frac{\omega_{0} /\left(\rho_{0} \tau\right)}{\left(\omega_{0}^{2}-c_{\gamma}^{2} k^{2}\right)^{2}+\omega_{0}^{2} / \tau^{2}}=-\frac{\pi}{2 \rho_{0} \omega_{0} c_{\gamma}}\left[\delta\left(\frac{\omega_{0}}{c_{\gamma}}-k\right)+\delta\left(\frac{\omega_{0}}{c_{\gamma}}+k\right)\right]
$$

Upon inserting the previous relation in equation (61) and integrating over the wave number $k$, we arrive at:

$$
\frac{1}{2 i}\left(T_{\alpha \beta}^{0}\left(\mathbf{k}_{a}, \mathbf{k}_{b}\right)-T_{\beta \alpha}^{0}\left(\mathbf{k}_{b}, \mathbf{k}_{a}\right)^{*}\right)=-\frac{\pi \omega_{0}}{2 \rho_{0}} \sum_{\gamma} \frac{1}{\left(c_{\gamma}\right)^{3}} \iint_{4 \pi} T_{\gamma \beta}^{0}\left(\frac{\omega_{0}}{c_{\gamma}} \hat{\mathbf{k}}, \mathbf{k}_{b}\right) T_{\gamma \alpha}^{0}\left(\frac{\omega_{0}}{c_{\gamma}} \hat{\mathbf{k}}, \mathbf{k}_{a}\right)^{*} d^{2} \hat{k}
$$


which is the form of the generalized optical theorem in a medium with infinitesimal absorption. In Equation (63), the symbol $d^{2} \hat{k}$ denotes the element of solid angle in wavenumber space. Again, off-shell terms of the $T$ matrix appear on both side of equation (63). On the right hand side, two arguments are on the shell $c_{\gamma}^{2} k^{2}=\omega_{0}^{2}$. Physically, off-shell terms are related to the physical situation where source and detection take place in the near field of the scatterer (Peierls, 1979). Thus the reconstruction of Green's function for arbitrary locations of the receivers in the medium imposes symmetry relations that pertain to the off-shell $T$-matrix. More examples of the optical theorem for electromagnetic and quantum waves can be found in the book of Newton (2002). If we now impose $\alpha=\beta$ and further restrict all wavenumbers to the shell $\omega_{0}$, we arrive at classical forms of the optical theorem:

$$
\operatorname{Im} T_{\alpha \alpha}^{0}\left(\frac{\omega_{0}}{c_{\alpha}} \hat{\mathbf{k}}_{a}, \frac{\omega_{0}}{c_{\alpha}} \hat{\mathbf{k}}_{a}\right)=-\frac{\pi \omega_{0}}{2 \rho_{0}} \sum_{\gamma} \frac{1}{c_{\gamma}^{3}} \iint_{4 \pi}\left|T_{\gamma \alpha}^{0}\left(\frac{\omega_{0}}{c_{\gamma}} \hat{\mathbf{k}}, \frac{\omega_{0}}{c_{\alpha}} \hat{\mathbf{k}}_{a}\right)\right|^{2} d^{2} \hat{k}
$$

As discussed in Peierls (1979), the on-shell matrix elements describe the angular dependence of the scattered waves in the far-field of the heterogeneity. The physical meaning of equation (64) for elastic waves will be further elucidated in section [6. It is important to keep in mind that all the relations obtained so far have been based on the Green's function reconstruction theorem. In the next section, we make the link with the scattering theory developed in section 4 .

\section{Generalized optical theorem: formal derivations}

In this section, we provide derivations of the various forms of optical theorems obtained within the framework of Green's function reconstruction. First, we note that Equation (64) which is the on-shell version of equation (63), can be obtained straightforwardly from the scattering theory developed in section (4):

$$
\begin{gathered}
\frac{1}{2 i}\left[T_{\alpha \beta}^{0}\left(\frac{\omega_{0}}{c_{\alpha}} \hat{\mathbf{k}}_{a}, \frac{\omega_{0}}{c_{\beta}} \hat{\mathbf{k}}_{b}\right)-T_{\beta \alpha}^{0}\left(\frac{\omega_{0}}{c_{\beta}} \hat{\mathbf{k}}_{b}, \frac{\omega_{0}}{c_{\alpha}} \hat{\mathbf{k}}_{a}\right)^{*}\right]= \\
\frac{1}{2 i}\left[\left\langle\alpha, \frac{\omega_{0}}{c_{\alpha}} \hat{\mathbf{k}}_{a}\left|T^{0}\right| \beta, \frac{\omega_{0}}{c_{\beta}} \hat{\mathbf{k}}_{b}\right\rangle-\left\langle\alpha, \frac{\omega_{0}}{c_{\alpha}} \hat{\mathbf{k}}_{a}\left|T^{0 \dagger}\right| \beta, \frac{\omega_{0}}{c_{\beta}} \hat{\mathbf{k}}_{b}\right\rangle\right] \\
=\frac{1}{2 i}\left[\left\langle\alpha, \frac{\omega_{0}}{c_{\alpha}} \hat{\mathbf{k}}_{a}\left|V\left(\omega_{0}^{2}\right)\right| \psi^{\beta}\left(\frac{\omega_{0}}{c_{\beta}} \hat{\mathbf{k}}_{b}\right)\right\rangle-\left\langle\psi^{\alpha}\left(\frac{\omega_{0}}{c_{\alpha}} \hat{\mathbf{k}}_{a}\right)\left|V\left(\omega_{0}^{2}\right)\right| \beta, \frac{\omega_{0}}{c_{\beta}} \hat{\mathbf{k}}_{b}\right\rangle\right] \\
=\frac{1}{2 i}\left[\left\langle\psi^{\alpha}\left(\frac{\omega_{0}}{c_{\alpha}} \hat{\mathbf{k}}_{a}\right)\left|V\left(\omega_{0}^{2}\right)\right| \psi^{\beta}\left(\frac{\omega_{0}}{c_{\beta}} \hat{\mathbf{k}}_{b}\right)\right\rangle-\left\langle\psi^{\alpha}\left(\frac{\omega_{0}}{c_{\alpha}} k^{\alpha} \hat{\mathbf{k}}_{a}\right)\left|V\left(\omega_{0}^{2}\right) G^{0}\left(\omega_{0}\right)^{\dagger} V\left(\omega_{0}^{2}\right)\right| \beta, \frac{\omega_{0}}{c_{\beta}} \hat{\mathbf{k}}_{b}\right\rangle\right] \\
-\frac{1}{2 i}\left[\left\langle\psi^{\alpha}\left(\frac{\omega_{0}}{c_{\alpha}} \hat{\mathbf{k}}_{a}\right)\left|V\left(\omega_{0}^{2}\right)\right| \psi^{\beta}\left(\frac{\omega_{0}}{c_{\beta}} \hat{\mathbf{k}}_{b}\right)\right\rangle+\left\langle\psi^{\alpha}\left(\frac{\omega_{0}}{c_{\alpha}} \hat{\mathbf{k}}_{a}\right)\left|V\left(\omega_{0}^{2}\right) G^{0}\left(\omega^{0}\right) V\left(\omega_{0}^{2}\right)\right| \psi^{\beta}\left(\frac{\omega_{0}}{c_{\beta}} \hat{\mathbf{k}}_{b}\right)\right\rangle\right] \\
=\frac{1}{2 i}\left[\left\langle\psi^{\alpha}\left(\frac{\omega_{0}}{c_{\alpha}} \hat{\mathbf{k}}_{a}\right)\left|V\left(\omega_{0}^{2}\right)\left(G^{0}\left(\omega^{0}\right)-G^{0}\left(\omega^{0}\right)^{\dagger}\right) V\left(\omega_{0}^{2}\right)\right| \psi^{\beta}\left(\frac{\omega_{0}}{c_{\beta}} \hat{\mathbf{k}}_{b}\right)\right\rangle\right] \\
=\frac{1}{2 i}\left[\left\langle\alpha, \frac{\omega_{0}}{c_{\alpha}} \hat{\mathbf{k}}_{a}\left|T^{0 \dagger}\left(G^{0}\left(\omega^{0}\right)-G^{0}\left(\omega^{0}\right)^{\dagger}\right) T^{0}\right| \beta, \frac{\omega_{0}}{c_{\beta}} \hat{\mathbf{k}}_{b}\right\rangle\right] \\
=-\frac{\pi \omega_{0}}{2 \rho_{0}} \sum_{\gamma} \frac{1}{\left(c_{\gamma}\right)^{3}} \iint_{4 \pi} T_{\gamma \beta}^{0}\left(\frac{\omega_{0}}{c_{\gamma}} \hat{\mathbf{k}}, \frac{\omega_{0}}{c_{\beta}} \hat{\mathbf{k}}_{b}\right) T_{\gamma \alpha}^{0}\left(\frac{\omega_{0}}{c_{\gamma}} \hat{\mathbf{k}}, \frac{\omega_{0}}{c_{\alpha}} \hat{\mathbf{k}}_{a}\right)^{*} d^{2} \hat{k}
\end{gathered}
$$

where the infinitesimal absorption limit $(\tau \rightarrow \infty)$ is understood throughout. The first equality follows from the definition of the $T^{0}$-operator. In the second equality, we make use of the relation (42) between Lippman-Schwinger eigenvectors and plane waves. The third equality follows from the LippmanSchwinger equation (41). The fifth equality makes again use of (42). We recover the generalized optical theorem (63) after inserting the spectral decomposition of the free space Green's function (59) in the sixth equality. Some on-shell forms of the optical theorem similar to (64)-65) have been previously 
derived in Lu et al. (2011) for a homogeneous and isotropic heterogeneity, and Budreck and Rose (1992) for a general heterogeneity. These formulas are less general than the fully off-shell expressions (61) and (63). The physical meaning of the formula (64) can be further elucidated by introducing the scattering amplitudes defined in (47). From the definition of the scattering cross-section as the ratio between the total energy scattered per unit time, normalized by the energy flux density of the incoming plane wave:

$$
\sigma_{\alpha}\left(\hat{\mathbf{k}}_{a}\right)=\sum_{\beta} \frac{\rho_{0} \omega_{0}^{2} c_{\beta}}{\rho_{0} \omega_{0}^{2} c_{\alpha}} \iint_{4 \pi}\left|f^{\beta \leftarrow \alpha}\left(\hat{\mathbf{k}}_{b}, \hat{\mathbf{k}}_{a}\right)\right|^{2} d^{2} \hat{k}_{b},
$$

we arrive at the following simple formula:

$$
\sigma_{\alpha}\left(\hat{\mathbf{k}}_{a}\right)=\frac{4 \pi}{k_{\alpha}} \operatorname{Im} f^{\alpha \leftarrow \alpha}\left(\hat{\mathbf{k}}_{a}, \hat{\mathbf{k}}_{a}\right),
$$

which has been derived by other authors in the past (Varatharajulu (1977); Dassios et al. (1987)). The physical meaning of equation (64) is now clear: it expresses the conservation of energy in the scattering process. Energy conservation is realized thanks to the interference between the incident wave and the wave scattered exactly in the forward direction in the far-field.

To prove more general versions of the optical theorem, we invoke the spectral decomposition of Green's function of the heterogeneous medium in terms of the Lippman-Schwinger eigenvectors. We make no attempt to justify rigorously this decomposition and proceed completely formally. Mathematical foundations of this approach can be found in the book of Reed and Simon (1979) for quantum waves, of Ramm (1986) for classical waves. A generalized eigenfunction expansion in terms of Lippman-Schwinger eigenvectors of the elastodynamic equation may also be found in the work of Budreck and Rose (1991). Making use of the eigenvectors of the operator $L^{0}+V\left(\omega_{0}^{2}\right)$ introduced in equation (38), we expand Green's function $G$ as follows:

$$
G\left(\omega_{0}\right)=\sum_{\gamma} \iint_{4 \pi} d^{2} \hat{k} \int_{0}^{+\infty} \frac{\left|\psi^{\gamma}(k \hat{\boldsymbol{k}})\right\rangle\left\langle\psi^{\gamma}(k \hat{\boldsymbol{k}})\right|}{\rho_{0}\left(\omega_{0}^{2}-c_{\gamma}^{2} k^{2}+i \omega_{0} / \tau\right)} k^{2} d k
$$

In the case of quantum waves, this decomposition is correct if the scattering potential does not create bound states. Using the formal definition of the $T^{\left(\omega_{0}^{2}\right)}$-operator (39) and the spectral representation (68), we find in the limit $(\tau \rightarrow \infty)$ :

$$
\begin{gathered}
\frac{1}{2 i}\left(T_{\alpha \beta}^{0}\left(\boldsymbol{k}_{a}, \boldsymbol{k}_{b}\right)-T_{\beta \alpha}^{0}\left(\boldsymbol{k}_{b}, \boldsymbol{k}_{a}\right)^{*}\right)=\frac{1}{2 i}\left(\left\langle\alpha, \boldsymbol{k}_{a}\left|V\left(\omega_{0}^{2}\right)\right| \beta, \boldsymbol{k}_{b}\right\rangle-\left\langle\alpha, \boldsymbol{k}_{a}\left|V\left(\omega_{0}^{2}\right)\right| \beta, \boldsymbol{k}_{b}\right\rangle\right)+ \\
\frac{1}{2 i}\left(\left\langle\alpha, \boldsymbol{k}_{a}\left|V\left(\omega_{0}^{2}\right) G\left(\omega_{0}\right) V\left(\omega_{0}^{2}\right)\right| \beta, \boldsymbol{k}_{b}\right\rangle-\left\langle\alpha, \boldsymbol{k}_{a}\left|V\left(\omega_{0}^{2}\right) G\left(\omega_{0}\right)^{\dagger} V\left(\omega_{0}^{2}\right)\right| \beta, \boldsymbol{k}_{b}\right\rangle\right) \\
=-\frac{\omega_{0}}{\rho_{0} \tau} \sum_{\gamma} \iint_{4 \pi} d^{2} \hat{k} \int_{0}^{+\infty} k^{2}\left\langle\alpha, \boldsymbol{k}_{a}\left|V\left(\omega_{0}^{2}\right) \frac{\left|\psi^{\gamma}(k \hat{\boldsymbol{k}})\right\rangle\left\langle\psi^{\gamma}(k \hat{\boldsymbol{k}})\right|}{\left.\left[\left(\omega_{0}^{2}-c_{\gamma}^{2} k^{2}\right)^{2}+\omega_{0}^{2} / \tau^{2}\right)\right]} V\left(\omega_{0}^{2}\right)\right| \beta, \boldsymbol{k}_{b}\right\rangle d k \\
=-\frac{\pi}{2 \rho_{0}} \sum_{\gamma} \iint_{4 \pi} d^{2} \hat{k} \int_{0}^{+\infty} \frac{k^{2}}{c_{\gamma} \omega_{0}}\left(\delta\left(k-\omega_{0} / c_{\gamma}\right)+\delta\left(k+\omega_{0} / c_{\gamma}\right)\right) \\
\times\left\langle\alpha, \boldsymbol{k}_{a}\left|T^{\omega_{0}^{2}}\left(c_{\gamma} k\right)\right| \gamma, k \hat{\boldsymbol{k}}\right\rangle\left\langle\gamma, k \hat{\boldsymbol{k}}\left|T^{\omega_{0}^{2}}\left(c_{\gamma} k\right)\right| \beta, \boldsymbol{k}_{b}\right\rangle d k \\
=-\frac{\pi \omega_{0}}{2 \rho_{0}} \sum_{\gamma} \frac{1}{c_{\gamma}^{3}} \iint_{4 \pi} d^{2} \hat{k} T_{\alpha \gamma}^{\omega_{0}^{2}}\left(\boldsymbol{k}_{a}, \frac{\omega_{0}}{c_{\gamma}} \hat{\mathbf{k}} ; c^{\gamma} k=\omega_{0}\right) T_{\beta \gamma}^{\omega_{0}^{2}}\left(\boldsymbol{k}_{b}, \frac{\omega_{0}}{c_{\gamma}} \hat{\mathbf{k}} ; c^{\gamma} k=\omega_{0}\right)^{*} \\
=-\frac{\pi \omega_{0}}{2 \rho_{0}} \sum_{\gamma} \frac{1}{c_{\gamma}^{3}} \iint_{4 \pi} d^{2} \hat{k} T_{\gamma \alpha}^{0}\left(\frac{\omega_{0}}{c_{\gamma}} \hat{\mathbf{k}}, \boldsymbol{k}_{a}\right)^{*} T_{\gamma \beta}^{0}\left(\frac{\omega_{0}}{c_{\gamma}} \hat{\mathbf{k}}, \boldsymbol{k}_{b}\right),
\end{gathered}
$$


which is identical to equation (63). In the derivation of equation (69), we made use of the limit (62) and of the reciprocity relations for the $T^{\left(\omega_{0}^{2}\right)}$-operator. In the third equality, we have made use of the Lippman-Schwinger eigenvectors introduced in equation (41). It is to be noted that the previous results are correct irrespective of the sign of $\omega_{0}$, which is important when dealing with classical waves.

\section{Application to a point-scatterer model}

We now illustrate the process of Green's function reconstruction with a simple example of a 3-D heterogeneous medium composed of a single delta-like scattering potential. To render multiple-scattering calculations easier, it is often convenient to introduce a point-scatterer model in the same spirit as in the scalar case (Margerin and Sato, 2011). Mathematically, point-scattering poses some serious difficulties (de Vries et al., 1998). Physically, the point scatterer model is obtained by regularizing the Born series for a scattering potential of the form:

$$
V\left(\omega_{0}^{2}\right)=\gamma\left(\omega_{0}^{2}\right) \mathcal{I}\left|\mathbf{x}_{0}\right\rangle\left\langle\mathbf{x}_{0}\right|
$$

where $\mathbf{x}_{0}$ is the location of the scattering center, and the physical significance of the parameter $\gamma\left(\omega_{0}^{2}\right)$ will be further discussed below. The scattering potential $V\left(\omega_{0}^{2}\right)$ has to be understood as a tensor product between two operators. The symbol $\mathcal{I}$ denotes the identity operator in polarization space. It simply leaves unchanged the vectorial part of the wavefunction. The symbol $\left|\mathbf{x}_{0}\right\rangle\left\langle\mathbf{x}_{0}\right|$ is a projection operator which picks out the value of the wavefunction exactly at position $\mathbf{x}_{0}$, hence the name "point-scatterer". We will find that the scattering potential $V\left(\omega_{0}^{2}\right)$ represents adequately a small scatterer with perfectly correlated perturbations of the density and $P$ and $S$ wave velocities according to: $\delta c_{p} / c_{p}=\delta c_{s} / c_{s}=-\delta \rho / 2 \rho$, $(\delta \lambda / \lambda=\delta \mu / \mu=0)$. No assumption on the smallness of the perturbation is made. In the representation $(2)$, the scattering potential writes

$$
V_{i j}\left(\mathbf{k}, \mathbf{k}^{\prime} ; \omega_{0}^{2}\right)=\frac{\gamma\left(\omega_{0}^{2}\right)}{(2 \pi)^{3}} e^{i \mathbf{x}_{0} \cdot\left(\mathbf{k}^{\prime}-\mathbf{k}\right)} \delta_{i j} .
$$

To evaluate the scattering properties of such a perturbation, we make use of the formal Born series for the $T$-operator of elastic waves:

$$
T^{0}=V\left(\omega_{0}^{2}\right)+V\left(\omega_{0}^{2}\right) G_{0}\left(\omega_{0}\right) V\left(\omega_{0}^{2}\right)+V\left(\omega_{0}^{2}\right) G_{0}\left(\omega_{0}\right) V\left(\omega_{0}^{2}\right) G_{0}\left(\omega_{0}\right) V\left(\omega_{0}^{2}\right)+\ldots
$$

and evaluate the matrix elements in the representation (2)

$$
\begin{array}{r}
\left\langle\mathbf{k}, i\left|T^{0}\right| j, \mathbf{k}^{\prime}\right\rangle=V_{i j}\left(\mathbf{k}, \mathbf{k}^{\prime} ; \omega_{0}^{2}\right)+\sum_{i_{1}} \iiint_{\mathbb{R}^{3}}\left\langle\mathbf{k}, i\left|V\left(\omega_{0}^{2}\right)\right| i_{1}, \mathbf{k}_{1}\right\rangle\left\langle i_{1}, \mathbf{k}_{1}\left|G^{0}\left(\omega_{0}\right) V\left(\omega_{0}^{2}\right)\right| j, \mathbf{k}^{\prime}\right\rangle d^{3} k_{1}+ \\
\sum_{i_{1}, i_{2}} \iiint_{\mathbb{R}^{6}}\left\langle\mathbf{k}, i\left|V\left(\omega_{0}^{2}\right)\right| i_{1}, \mathbf{k}_{1}\right\rangle\left\langle\mathbf{k}_{1}, i_{1}\left|G^{0}\left(\omega_{0}\right) V\left(\omega_{0}^{2}\right)\right| i_{2}, \mathbf{k}_{2}\right\rangle\left\langle i_{2}, \mathbf{k}_{2}\left|G^{0}\left(\omega_{0}\right) V\left(\omega_{0}^{2}\right)\right| j, \mathbf{k}^{\prime}\right\rangle d^{3} k_{1} d^{3} k_{2} \\
+\cdots
\end{array}
$$

The need for regularization stems from the fact that repeated visits of the same scatterer involve evaluations of the free space Green's function for coincident source and receiver, which clearly diverges. We now show that for a scattering potential of the form (70), the Born series (72) can be summed analytically after the return Green's function is made finite. To make sense of expression (73), we need to evaluate the matrix elements of the operator $G_{0}\left(\omega_{0}\right) V\left(\omega_{0}^{2}\right)$ :

$$
\begin{aligned}
\left\langle\mathbf{k}, i\left|G^{0}\left(\omega_{0}\right) V\left(\omega_{0}\right)\right| j, \mathbf{k}^{\prime}\right\rangle= & \sum_{i_{1}} \iiint_{\mathbb{R}^{3}}\left\langle\mathbf{k}, i\left|G^{0}\left(\omega_{0}\right)\right| i_{1}, \mathbf{k}_{1}\right\rangle\left\langle i_{1}, \mathbf{k}_{1}\left|V\left(\omega_{0}^{2}\right)\right| j, \mathbf{k}^{\prime}\right\rangle d^{3} k_{1} \\
= & \frac{\gamma\left(\omega_{0}^{2}\right) e^{i \mathbf{x}_{0} \cdot\left(\mathbf{k}^{\prime}-\mathbf{k}\right)}}{(2 \pi)^{3}} \\
& \times\left(\frac{\hat{k}_{i} \hat{k}_{j}}{\rho_{0}\left(\omega_{0}^{2}-\left(c_{p} k\right)^{2}+i \omega_{0} / \tau\right)}+\frac{\delta_{i j}-\hat{k}_{i} \hat{k}_{j}}{\rho_{0}\left(\omega_{0}^{2}-\left(c_{s} k\right)^{2}+i \omega_{0} / \tau\right)}\right),
\end{aligned}
$$


where we have made use of equations (14) and (71). Reporting expression (74) in the first integral of Equation (73) we obtain:

$$
\begin{aligned}
I_{1} & =\frac{\gamma\left(\omega_{0}^{2}\right) e^{i \mathbf{x}_{0} \cdot\left(\mathbf{k}^{\prime}-\mathbf{k}\right)}}{(2 \pi)^{3}} \iiint_{\mathbb{R}^{3}}\left(\frac{\hat{\mathbf{k}}_{1} \hat{\mathbf{k}}_{1}}{\rho_{0}\left(\omega_{0}^{2}-\left(c_{p} k_{1}\right)^{2}+i \omega_{0} / \tau\right)}+\frac{\mathcal{I}-\hat{\mathbf{k}}_{1} \hat{\mathbf{k}}_{1}}{\rho_{0}\left(\omega_{0}^{2}-c_{s}^{2} k_{1}^{2}+i \omega_{0} / \tau\right)}\right) d^{3} k_{1} \\
& =\frac{\gamma\left(\omega_{0}^{2}\right) e^{i \mathbf{x}_{0}\left(\mathbf{k}^{\prime}-\mathbf{k}\right)}}{(2 \pi)^{3}} \frac{\mathcal{I} 4 \pi \gamma\left(\omega_{0}^{2}\right)}{3(2 \pi)^{3}}\left(\int_{0}^{\infty} \frac{k_{1}^{2} d k_{1}}{\rho_{0}\left(\omega_{0}^{2}-c_{p}^{2} k_{1}^{2}+i \omega_{0} / \tau\right)}+2 \int_{0}^{\infty} \frac{k_{1}^{2} d k_{1}}{\rho_{0}\left(\omega_{0}^{2}-c_{s}^{2} k_{1}^{2}+i \omega_{0} / \tau\right)}\right),
\end{aligned}
$$

where we have used:

$$
\iint_{4 \pi} \hat{\mathbf{k}}_{1} \hat{\mathbf{k}}_{1} d^{2} \hat{k}_{1}=\frac{4 \pi \mathcal{I}}{3}
$$

Similarly, for the second integral of equation (72), one obtains:

$$
I_{2}=\frac{\gamma\left(\omega_{0}^{2}\right)}{(2 \pi)^{3}} e^{i \mathbf{x}_{0}\left(\mathbf{k}^{\prime}-\mathbf{k}\right)}\left[\frac{\mathcal{I} 4 \pi \gamma\left(\omega_{0}^{2}\right)}{3(2 \pi)^{3}}\left(\int_{0}^{\infty} \frac{k_{1}^{2} d k_{1}}{\rho_{0}\left(\omega_{0}^{2}-c_{p}^{2} k_{1}^{2}+i \omega_{0} / \tau\right)}+2 \int_{0}^{\infty} \frac{k_{1}^{2} d k_{1}}{\rho_{0}\left(\omega_{0}^{2}-c_{s}^{2} k_{1}^{2}+i \omega_{0} / \tau\right)}\right)\right]^{2} .
$$

From equations (75)-(77), we infer that the Born series is in fact a simple geometric series. The last integrals in (75)-(77) diverge and need to be regularized. We proceed as in van Rossum and Nieuwenhuizen (1999), by introducing a large momentum cut-off to avoid divergence of the real part of Green's function. Let us illustrate this procedure for the $P$ modes. We rewrite the first integral on the right-hand side of equation (77) as follows:

$$
\begin{aligned}
J & =\frac{1}{\rho_{0} c_{p}^{2}} \int_{0}^{\infty} \frac{k_{1}^{2} d k_{1}}{k_{p}^{2}-k_{1}^{2}+i k_{p} / l_{p}} \\
& =\frac{1}{\rho_{0} c_{p}^{2}} \int_{0}^{\infty} k_{1}^{2} d k_{1}\left[\frac{1}{\left(k_{p}^{2}-k_{1}^{2}+i k_{p} / l_{p}\right)}+\frac{1}{k_{1}^{2}}\right]-\frac{1}{\rho_{0} c_{p}^{2}} \int_{0}^{\infty} d k_{1},
\end{aligned}
$$

where $k_{p}=\omega_{0} / c_{p}$ and $l_{p}=c_{p} \tau$. Note that terms of order $1 / l_{p}^{2}$ have been neglected in the numerator, which implies that absorption is sufficiently small. In the first integral, we have subtracted out the divergent part. In the second integral, we introduce a large momentum cut-off with a scale length $a$ to obtain:

$$
\begin{aligned}
J & \approx \frac{1}{\rho_{0} c_{p}^{2}} \int_{0}^{\infty} \frac{\left(k_{p}+i / 2 l_{p}\right)^{2} d k_{1}}{k_{p}^{2}-k_{1}^{2}+i k_{p} / l_{p}}-\frac{1}{\rho_{0} c_{p}^{2}} \int_{0}^{1 / a} d k_{1} \\
& \approx-\frac{1}{\rho_{0} c_{p}^{2}}\left(\frac{1}{a}-\frac{\pi}{4 l_{p}}+i \frac{\pi k_{p}}{2}\right),
\end{aligned}
$$

where $k_{p}=\omega_{0} / c_{p}$. Combining equations (75)-(77) and (79), one may rewrite the formal series expression of the $T^{0}$-matrix given by (72) in the form:

$$
T_{i j}^{0}\left(\mathbf{k}, \mathbf{k}^{\prime}\right)=\frac{e^{i \mathbf{x}_{0}\left(\mathbf{k}^{\prime}-\mathbf{k}\right)} t\left(\omega_{0}\right) \delta_{i j}}{(2 \pi)^{3}}
$$

where the scalar $t\left(\omega_{0}\right)$-matrix can be expressed as:

$$
t\left(\omega_{0}\right)=\frac{\gamma\left(\omega_{0}^{2}\right)}{1-\gamma\left(\omega_{0}^{2}\right) U\left(\omega_{0}\right)}
$$


and we have introduced an effective potential:

$$
\begin{aligned}
U\left(\omega_{0}\right) & =-\frac{1}{6 \rho_{0} \pi^{2}}\left[\kappa+i \pi\left(\frac{\omega_{0}}{2 c_{p}^{3}}+\frac{\omega_{0}}{c_{s}^{3}}\right)\right] \\
\kappa & =\frac{1}{a}\left(\frac{2}{c_{s}^{2}}+\frac{1}{c_{p}^{2}}\right)-\frac{\pi}{2 \tau}\left(\frac{1}{2 c_{p}^{3}}+\frac{1}{c_{s}^{3}}\right)
\end{aligned}
$$

where the length $a$ stems from the large momentum cut-off. The result (81) is valid irrespective of the sign of $\omega_{0}$. Equations (80)-(81) offer a generalization of the point-scattering model to vector elastic waves. The regularization procedure is not unique as illustrated by de Vries et al. (1998) for scalar and electromagnetic waves. The most important point is the preservation of the local imaginary part of the free space Green's function:

$$
\operatorname{Im} G_{i j}^{0}(\mathbf{x}, \mathbf{x})=-\frac{\delta_{i j}}{4 \pi \rho_{0}}\left(\frac{\omega}{3 c_{p}^{3}}+\frac{2 \omega}{3 c_{s}^{3}}\right),
$$

which is proportional to the density of states, i.e. the number of modes per unit volume and frequency. We note that the imaginary part of the free space Green's function at $(\mathbf{x}, \mathbf{x})$ is an equipartition mixture of $P$ and $S$ waves and does not depend on the absorption time $\tau$. The result (81) for the $t$-matrix is valid for a small, finite amount of uniform absorption inside the medium. Note that because the absorbing properties of the scatterer match those of the embedding matrix the scattering potential is real.

To analyze the properties of our point scatterer, we make use of the relation (47) providing the relation between the scattering amplitude and the $T^{0}$-operator. The scattering pattern is obtained by sandwiching the $T^{0}$-operator between two plane waves, i.e. we take the matrix elements of $T^{0}$ in representation (3).

$$
f^{\alpha \leftarrow \beta}\left(\hat{\mathbf{k}}_{a} ; \hat{\mathbf{k}}_{b}\right) \propto\left\langle\mathbf{k}_{a}, \alpha\left|T^{0}\right| \mathbf{k}_{b}, \beta\right\rangle \propto\left\langle\mathbf{p}^{\alpha}|\mathcal{I}| \hat{\mathbf{q}}^{\beta}\right\rangle=\hat{\mathbf{p}}^{\alpha} \cdot \hat{\mathbf{q}}^{\beta},
$$

where $\hat{\mathbf{p}}^{\alpha}, \hat{\mathbf{q}}^{\beta}$ denote the polarization vector of the scattered and incident wave, respectively. Let us specialize the last result by introducing a spherical coordinate system where the polar angle $\theta$ is measured from the axis pointing in the direction of the incident wave with wavevector $\mathbf{k}_{b}$. For the different scattering type, we obtain the following angular dependence: (1) $P$ to $P: \cos \theta ;(2) P$ to $S V$ and $S V$ to $P: \sin \theta$; (3) $S V$ to $S V: \cos \theta$, (4) $S H$ to $S H$ : no angular dependence (in and out polarization vectors are parallel), where $S V$ and $S H$ denote transverse polarizations in and perpendicular to the scattering plane, respectively. All other coupling terms are zero. These results agree with the scattering pattern for a pure density perturbation (constant Lamé parameters) in the low-frequency or Rayleigh regime. The scattering pattern is anisotropic but non-preferential, i.e., there is equal amount of forward and backward scattering. This property is typical of a scatterer whose size is much smaller than the wavelength. There is no mode conversion in the forward direction as required by symmetry consideration. Reporting expression (80) in the usual form of the optical theorem (67), we find the following relations for the scattering cross-sections in terms of the $t$-matrix:

$$
\begin{aligned}
\sigma_{p} & =-\frac{1}{\rho_{0} c_{p}} \operatorname{Im} \frac{t}{\omega_{0}} \\
\sigma_{s} & =-\frac{1}{\rho_{0} c_{s}} \operatorname{Im} \frac{t}{\omega_{0}},
\end{aligned}
$$

which implies:

$$
c_{p} \sigma_{p}=c_{s} \sigma_{s}
$$

The relation (87) is valid for a point scatterer but not generally true. Finally, let us identify the parameter $\gamma\left(\omega_{0}^{2}\right)$ with $-\delta \rho \omega_{0}^{2} \mathcal{V}$, with $\mathcal{V}$ the volume of the scatterer and $\delta \rho$ the density contrast between the scatterer and the matrix. By substituting expression (81) into expression (86a), and taking the limit $\omega_{0} \rightarrow 0$, we find:

$$
\sigma_{p}=\frac{2 \mathcal{V}}{9}\left(\frac{1}{2 c_{p}^{3}}+\frac{1}{c_{s}^{3}}\right)\left(\frac{\delta \rho}{\rho_{0}}\right)^{2} \frac{\omega_{0}^{4} r^{3}}{c_{p}},
$$


with $r$ the scatterer radius. These results concur with previous analytical solutions of the scattering problem for a spherical inclusion in the Rayleigh regime (Korneev and Johnson, 1993). We have therefore defined a physically meaningful point-scatterer for elastic waves. It is important to note that relation (87) pertains to a localized perturbation of the density at fixed Lamé parameters $\lambda$ and $\mu$.

The final step of our investigation of the point scatterer concerns the verification of the complete identity (61). On the right-hand side, we find:

$$
\frac{1}{2 i}\left(T_{\alpha \beta}^{0}\left(\mathbf{k}_{a}, \mathbf{k}_{b}\right)-T_{\beta \alpha}^{0}\left(\mathbf{k}_{b}, \mathbf{k}_{a}\right)^{*}\right)=\frac{\hat{\mathbf{p}}^{\alpha} \cdot \hat{\mathbf{q}}^{\beta} e^{i \mathbf{x}_{0} \cdot\left(\mathbf{k}_{b}-\mathbf{k}_{a}\right)} \operatorname{Im} t\left(\omega_{0}\right)}{(2 \pi)^{3}}
$$

The left-hand side can be calculated as follows:

$$
\begin{aligned}
& -\frac{\omega_{0}}{\rho_{0} \tau} \sum_{\gamma} \iiint \frac{T_{\gamma \beta}^{0}\left(\mathbf{k}, \mathbf{k}_{b}\right) T_{\gamma \alpha}^{0}\left(\mathbf{k}, \mathbf{k}_{a}\right)^{*}}{\left(\omega_{0}^{2}-\left(c_{\gamma} k\right)^{2}\right)^{2}+\omega_{0}^{2} / \tau^{2}} d^{3} k= \\
& -\frac{\omega_{0}}{\rho_{0} \tau(2 \pi)^{6}} \sum_{\gamma} \iiint \frac{\left|t\left(\omega_{0}\right)\right|^{2} e^{i \mathbf{x}_{0} \cdot\left(\mathbf{k}_{b}-\mathbf{k}_{a}\right)}\left\langle\hat{\mathbf{p}}^{\alpha} \mid \gamma, \mathbf{k}\right\rangle\left\langle\gamma, \mathbf{k} \mid \hat{\mathbf{q}}^{\beta}\right\rangle}{\left(\omega_{0}^{2}-\left(c_{\gamma} k\right)^{2}\right)^{2}+\omega_{0}^{2} / \tau^{2}} d^{3} k \\
& =-\frac{4 \pi \omega_{0}\left|t\left(\omega_{0}\right)\right|^{2} \hat{\mathbf{p}}^{\alpha} \cdot \hat{\mathbf{q}}^{\beta}}{3 \rho_{0} \tau(2 \pi)^{6}} \sum_{\gamma} \int_{0}^{+\infty} \frac{k^{2} d k}{\left(\omega_{0}^{2}-\left(c_{\gamma} k\right)^{2}\right)^{2}+\omega_{0}^{2} / \tau^{2}} \\
& =\frac{-\omega_{0}\left|t\left(\omega_{0}\right)\right|^{2} \hat{\mathbf{p}}^{\alpha} \cdot \hat{\mathbf{q}}^{\beta}}{(2 \pi)^{3} 6 \pi \rho_{0}}\left(\frac{1}{c_{s}^{3}}+\frac{1}{2 c_{p}^{3}}\right),
\end{aligned}
$$

a result valid in both absorbing (finite $\tau$ ) and non-absorbing media $(\tau \rightarrow \infty)$. From equation (84), we deduce a relation analog to the scalar case (van Rossum and Nieuwenhuizen, 1999):

$$
\operatorname{Im} t\left(\omega_{0}\right) \mathcal{I}=\left|t\left(\omega_{0}\right)\right|^{2} \operatorname{Im} G^{0}\left(\mathbf{x}, \mathbf{x} ; \omega_{0}\right) .
$$

A straightforward calculation shows that relation (91) is indeed verified by the $t$-matrix obtained from the regularization procedure. With this simple example we have therefore illustrated the relation between the reconstruction of the elastic Green's function and the conservation of energy in scattering. These results can be generalized to more complex multiple-scattering media. Such developments will be presented elsewhere.

\section{Discussion and Conclusion}

The reconstruction of Green's function of a homogeneous elastic medium from random noise sources was previously studied by Sánchez-Sesma and Campillo (2006). These authors used a decomposition of the random wavefield into a sum of uncorrelated plane $P$ and $S$ waves at equipartition. In this paper, we gave a precise mathematical formulation of the equipartition condition in equation (34). Using the Dirac calculus, we can show the complete equivalence between the equipartition state and an excitation of the system by randomly oriented and homogeneously distributed uncorrelated forces. Noting the relations:

$$
\begin{aligned}
f_{l}\left(\mathbf{x}_{1} ; \omega_{1}\right) f_{k}\left(\mathbf{x}_{0} ; \omega_{0}\right)^{*} & =\left\langle l, \mathbf{x}_{1} \mid f\left(\omega_{1}\right)\right\rangle\left\langle f\left(\omega_{0}\right) \mid k, \mathbf{x}_{0}\right\rangle \\
\tilde{f}_{\beta}\left(\mathbf{k}_{1} ; \omega_{1}\right) \tilde{f}_{\alpha}\left(\mathbf{k}_{0} ; \omega_{0}\right)^{*} & =\left\langle\beta, \mathbf{k}_{1} \mid f\left(\omega_{1}\right)\right\rangle\left\langle f\left(\omega_{0}\right) \mid \alpha, \mathbf{k}_{0}\right\rangle,
\end{aligned}
$$




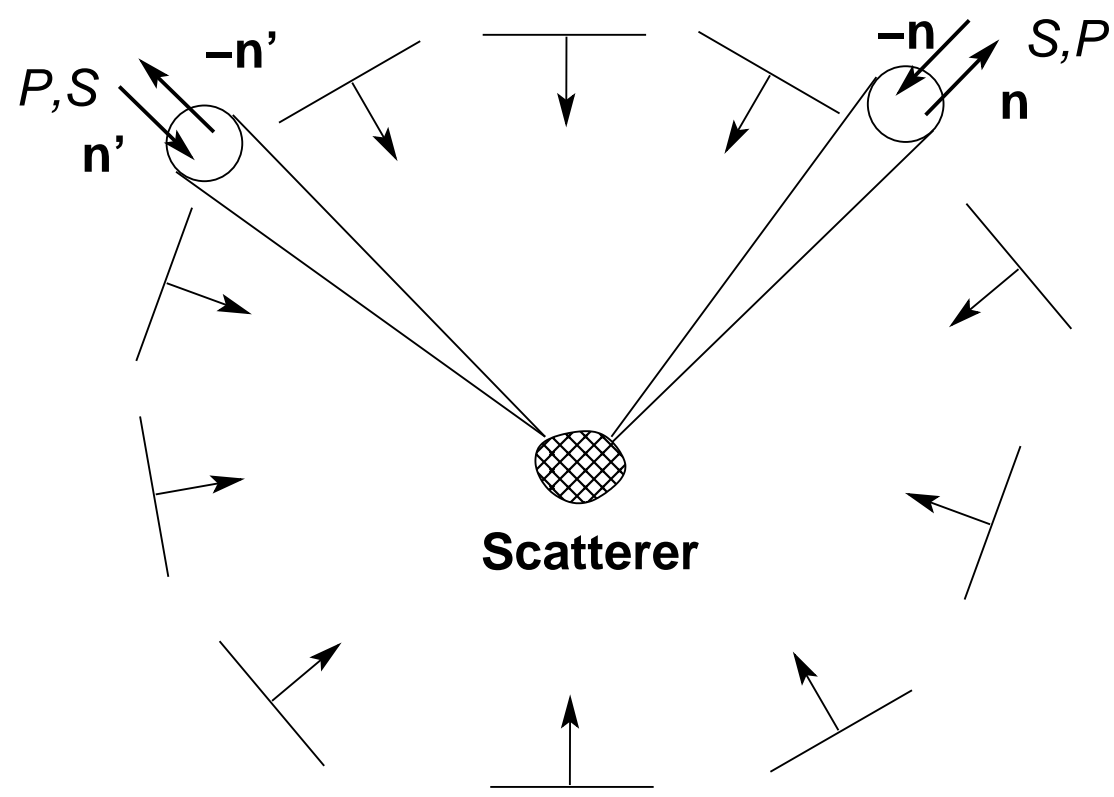

Figure 3: Energy balance of a random wavefield in a homogeneous isotropic elastic medium containing a scatterer. The scatterer is illuminated by uncorrelated plane $P$ and $S$ waves coming from all possible space directions. The total energy carried by $P$ waves increases because an $S$ wave propagating in direction $\mathbf{n}^{\prime}$ can be converted into a $P$ wave propagating in direction $\mathbf{n}$. The reciprocal situation shows a $P$ wave propagating in direction $-\mathbf{n}$ and converted into an $S$ wave propagating in direction $-\mathbf{n}^{\prime}$. This scattering process entails a decrease of the total $P$ wave energy. For an incident wavefield at equipartition the two contributions cancel exactly. The same argument applies to the total $S$ energy. 
we obtain:

$$
\begin{aligned}
\left.\left\langle\tilde{f}_{\beta}\left(\mathbf{k}_{1} ; \omega_{1}\right) \tilde{f}_{\alpha}\left(\mathbf{k}_{0} ; \omega_{0}\right)^{*}\right\rangle\right\rangle= & \\
\sum_{k, l} \iiint_{\mathbb{R}^{6}} & \left\langle\left(\left\langle\beta, \mathbf{k}_{1} \mid l, \mathbf{x}_{1}\right\rangle\left\langle l, \mathbf{x}_{1} \mid f\left(\omega_{1}\right)\right\rangle\left\langle f\left(\omega_{0}\right) \mid k, \mathbf{x}_{0}\right\rangle\left\langle k, \mathbf{x}_{0} \mid \alpha, \mathbf{k}_{0}\right\rangle\right)\right\rangle d^{3} x_{0} d^{3} x_{1} \\
= & 2 \pi S\left(\omega_{0}\right) \delta\left(\omega_{0}-\omega_{1}\right) \\
& \times \sum_{k, l} \iiint_{\mathbb{R}^{6}} \delta\left(\mathbf{x}_{0}-\mathbf{x}_{1}\right) \delta_{k l}\left(\left\langle\beta, \mathbf{k}_{1} \mid k, \mathbf{x}_{0}\right\rangle\left\langle l, \mathbf{x}_{1} \mid \alpha, \mathbf{k}_{0}\right\rangle\right) d^{3} x_{0} d^{3} x_{1} \\
= & 2 \pi S\left(\omega_{0}\right) \delta\left(\omega_{0}-\omega_{1}\right) \sum_{k} \iiint_{\mathbb{R}^{3}}\left\langle\beta, \mathbf{k}_{1} \mid k, \mathbf{x}_{0}\right\rangle\left\langle k, \mathbf{x}_{0} \mid \alpha, \mathbf{k}_{0}\right\rangle d^{3} x_{0} \\
= & 2 \pi S\left(\omega_{0}\right) \delta\left(\omega_{0}-\omega_{1}\right) \delta_{\alpha \beta} \delta\left(\mathbf{k}_{0}-\mathbf{k}_{1}\right)
\end{aligned}
$$

Equation (93), demonstrates that from the point of view of correlations, a white noise distribution of random forces and a wavefield at equipartition are equivalent. We may also illustrate this point with physical arguments. First we note that the radiation of energy by a single force can be evaluated from the far-field solutions. For the $P$-wave radiation, we multiply $r^{2} \rho_{0} \omega_{0}^{2} c_{p}$ by the squared modulus of Green's function $g^{p}\left(\mathbf{r} ; \omega_{0}\right)$ as given by Equation (10b). The procedure is the same for the $S$ wave radiation, except for the substitution $c_{p} \rightarrow c_{s}$. It follows that the ratio between the total $S$ - and $P$ - energy radiated by a force is $2\left(c_{p} / c_{s}\right)^{3}$, which rigorously coincides with the ratio of the equipartition state. A homogeneous distribution of randomly oriented forces will therefore generate $P$ and $S$ waves propagating in all possible directions with a local energy density of $P$ and $S$ waves satisfying the equipartition relation $E^{S} / E^{P}=2 c_{p}^{3} / c_{s}^{3}$. Thus we find that the force distribution (12) guarantees the existence of the equipartition state and the reconstruction of Green's function. It is important to note that the model of absorption that we have developed implies equality of the intrinsic quality factor between $P$ and $S$ waves. This assumption may not always be fulfilled in practice. If the absorption times of $P$ and $S$ waves are different, the $P$-to- $S$ energy ratio differs from that of the equipartition state and Green's function reconstruction will be imperfect.

The requirement of equipartition for the successful reconstruction of Green's function of a heterogeneous medium has been widely noted. Derode et al. (2003) have studied numerically and theoretically the reconstruction of Green's function of a heterogeneous, open medium illuminated by either random or deterministic sources. They gave a criterion based on time-reversal arguments to discuss the optimal arrangement of the sources. The reconstruction of Green's function of a heterogeneous medium illuminated by an incident random wavefield at equipartition has been considered theoretically by Weaver and Lobkis (2004) for scalar waves. Sánchez-Sesma et al. (2006) have demonstrated that when a cylindrical scatterer is illuminated by random plane $P$ and $S$ waves at equipartition in 2-D, the cross-correlation of the wavefield allows the reconstruction of the complete Green's function, including the waves scattered by the cylinder. In the case of a distribution of sources on a remote surface enclosing the receivers and a 3-D isotropic and homogeneous heterogeneity, Lu et al. (2011) showed that the wavefield incident on the scatterer must obey an equipartition relation. Their derivation supposes that the medium is free from absorption. In this work, we have generalized these results to an arbitrary, localized heterogeneity and have given an application to a point-scatterer. To make the link with previous investigations, we now consider the impact of a localized scatterer on the equipartition state. In Figure 3, we present a detailed balance of the energy carried by each wave mode to show that equipartition is globally preserved in a scattering system. Let us consider a bundle of plane $P$ waves coming from space direction $\mathbf{n}^{\prime}$ with mean squared amplitude $\left\langle\left\langle\left|A^{P}\left(\mathbf{n}^{\prime}\right)\right|^{2}\right\rangle\right\rangle$. Upon scattering, the $P$ waves will be mode converted into $S$ waves propagating into direction $\mathbf{n}$. Reciprocally, we may consider incident $S$ waves propagating in direction $-\mathbf{n}$ with mean-squared amplitude $\left\langle\left\langle\left|A^{S}(-\mathbf{n})\right|^{2}\right\rangle\right\rangle$ and mode converted into $P$ waves propagating in direction 
$-\mathbf{n}^{\prime}$. Let us now consider the balance of energy carried by $P$ waves in the scattering process:

$$
\begin{aligned}
& \frac{d E^{P}}{d t}=-\rho_{0} \omega_{0}^{2} c_{s} \iint_{4 \pi \times 4 \pi} d^{2} n\left\langle\left\langle\left|A^{P}\left(\mathbf{n}^{\prime}\right)\right|^{2}\right\rangle\right\rangle\left(\left|f^{S_{1} \leftarrow P}\left(\mathbf{n}, \mathbf{n}^{\prime}\right)\right|^{2}+\left|f^{S_{2} \leftarrow P}\left(\mathbf{n}, \mathbf{n}^{\prime}\right)\right|^{2}\right) d^{2} n^{\prime} \\
&+\rho_{0} \omega_{0}^{2} c_{p} \iint_{4 \pi \times 4 \pi} d^{2} n\left(\left\langle\left\langle\left|A^{S_{1}}(-\mathbf{n})\right|^{2}\right\rangle\right\rangle\left|f^{P \leftarrow S_{1}}\left(-\mathbf{n}^{\prime},-\mathbf{n}\right)\right|^{2}\right. \\
&\left.\left\langle\left\langle\left|A^{S_{2}}(-\mathbf{n})\right|^{2}\right\rangle\right\rangle\left|f^{P \leftarrow S_{2}}\left(-\mathbf{n}^{\prime},-\mathbf{n}\right)\right|^{2}\right) d^{2} n^{\prime},
\end{aligned}
$$

where $E^{P}$ denotes the total energy carried by $P$ waves. In equation (94), the integrals are performed over all possible space directions $\mathbf{n}$ and $\mathbf{n}^{\prime}$. The first term represents the rate of energy conversion from $P$ waves to $S$ waves in the scattering process. This contributes to a decrease to the total $P$ energy, hence the minus sign. The second term corresponds to the reciprocal process where $P$ energy increases because of the $S$ to $P$ mode conversions. For the description of the shear wave motion, we have chosen a basis of linear polarizations $\left\{S_{1}, S_{2}\right\}$ as illustrated in Figure 2 Other bases such as left-handed and righthanded circular polarizations are popular in optics. For a wavefield at equipartition the mean squared amplitudes are independent of the vectors $\mathbf{n}$ and $\mathbf{n}^{\prime}$ and satisfy the relations: $c_{s}^{3}\left\langle\left\langle\left|A^{S_{1}}\right|^{2}\right\rangle\right\rangle=c_{p}^{3}\left\langle\left\langle\left|A^{P}\right|^{2}\right\rangle\right\rangle$, $\left\langle\left\langle\left|A^{S_{1}}\right|^{2}\right\rangle\right\rangle=\left\langle\left\langle\left|A^{S_{2}}\right|^{2}\right\rangle\right\rangle$. Because of the fundamental reciprocity relations of conversion scattering derived in equation (50), we find that the total energy carried by $P$ waves is constant, i.e. $d E^{P} / d t=0$. This implies that equipartition is globally preserved by the introduction of a scatterer in the medium. This simple argument highlights again the link between Green's function reconstruction and equipartition.

In this paper, the link between symmetry relations in scattering and the reconstruction of Green's function from random noise sources has been generalized to the case of elastic waves (see Snieder et al. (2008); Snieder and Fleury (2010); Margerin and Sato (2011) for the acoustic case). Using the Dirac calculus, a number of previously known symmetry and reciprocity relations for the scattering amplitude have been derived in a systematic way (Varatharajulu, 1977; Dassios et al., 1987; Aki, 1992). Based on the general Green's function reconstruction formula for stationary random noise sources, we have obtained a general form of optical theorem valid in both near and far-field, which incorporates the effect of a finite amount of absorption. We also show that the generalized optical theorem can be established from the scattering formalism in the limit of infinitesimal absorption. Thus, the correlation properties of random wavefields offer an alternative approach to study scattering phenomena in heterogeneous media. The reconstruction of Green's function in 3-D is illustrated with the simple case of a point-like heterogeneity embedded in an isotropic matrix. Based on a regularization of the return free space Green's function, we are able to sum the Born series for such a defect. Usual results of the formal boundary value problem for a small scatterer with an arbitrary density contrast are recovered in the low frequency limit. The point scatterer satisfies the generalized optical theorem, which in turn implies the reconstruction of Green's function in the presence of random distribution of noise sources. The point scattering model offers an attractive approach to tackle more complex multiple-scattering media. Such developments will be the topic of a separate publication.

\section{Acknowledgments}

We would like to thank K. Wapenaar for his detailed review, and for his suggestions to improve the clarity of the manuscript. We thank an anonymous referee for drawing our attention to recent references on Green's function retrieval. This work was supported by a joint program of the Japanese society for the promotion of science (Japan) and the Centre national de la recherche scientifique (France).

\section{References}

Aki, K. (1992). "Scattering conversions P to S versus S to P", Bull. Seismol. Soc. Am 82, 1969-1972.

Ben-Menahem, A. and Singh, S. (1998). Seismic waves and sources (Dover Publications, New York), $29-32$. 
Brenguier, F., Campillo, M., Hadziioannou, C., Shapiro, N. M., Nadeau, R. M., and Larose, E. (2008a). "Postseismic Relaxation Along the San Andreas Fault at Parkfield from Continuous Seismological Observations", Science 321, 1478-1481.

Brenguier, F., Shapiro, N. M., Campillo, M., Ferrazzini, V., Duputel, Z., Coutant, O., and Nercessian, A. (2008b). "Towards forecasting volcanic eruptions using seismic noise", Nature Geoscience 1, 126-130.

Budreck, D. and Rose, J. (1991). "Elastodynamic completeness relations for scattered wavefields", SIAM Journal on Applied Mathematics 1568-1584.

Budreck, D. and Rose, J. (1992). "A Newton-Marchenko equation and generalized optical theorem for elastodynamics", J. Math. Phys. 33, 2903-2915.

Dassios, G., Kiriaki, K., and Polyzos, D. (1987). "On the scattering amplitudes for elastic waves", Zeitschrift für Angewandte Mathematik und Physik (ZAMP) 38, 856-873.

de Vries, P., van Coevorden, D., and Lagendijk, A. (1998). "Point scatterers for classical waves", Rev. Mod. Phys. 70, 447-466.

Derode, A., Larose, E., Tanter, M., de Rosny, J., Tourin, A., Campillo, M., and Fink, M. (2003). "Recovering the Greens function from field-field correlations in an open scattering medium (L)", J. Acous. Soc. Am. 113, 2973-2976.

Economou, E. N. (2006). Green's Functions in Quantum Physics (Springer, Berlin), 477 p.

Korneev, V. and Johnson, L. (1993). "Scattering of elastic waves by a spherical inclusion II. Limitations of asymptotic solutions", Geophys. J. Int. 115, 251-263.

Lu, L., Ding, Z., Zeng, R., and He, Z. (2011). "Retrieval of Green's function and generalized optical theorem for the scattering of complete dyadic fields", J. Acoust. Soc. Am. 129, 1935-1944.

Margerin, L. and Sato, H. (2011). "Reconstruction of multiply-scattered arrivals from the crosscorrelation of waves excited by random noise sources in a heterogeneous dissipative medium", Wave Motion 48, 146-160.

Messiah, A. (1999). Quantum mechanics (2 volumes bound as one) (Dover, New York), 549-551.

Newton, R. (2002). Scattering theory of waves and particles (Dover Publications, New York) 745 p.

Peierls, S. (1979). Surprises in theoretical physics (Princeton University Press, Princeton), 127-132.

Poupinet, G., Ellsworth, W., and Frechet, J. (1984). "Monitoring velocity variations in the crust using earthquake doublets: an application to the Calaveras fault, California", J. geophys. Res 89, 5719-5731.

Poupinet, G., Got, J.-L., and Brenguier, F. (2008). Earth Heterogeneity and Scattering Effects on Seismic Waves, volume 50 of Advances in Geophysics, chapter Monitoring temporal variations of physical properties in the crust by cross-correlating the waveforms of seismic doublets, 374-399 (Academic Press, New York).

Ramirez, A. and Weglein, A. (2009). "Green's theorem as a comprehensive framework for data reconstruction, regularization, wavefield separation, seismic interferometry, and wavelet estimation: A tutorial", Geophysics 74, W35-W62.

Ramm, A. (1986). Scattering by obstacles (Kluwer Academic Publishers, Dordrecht, Holland), 444 p.

Reed, M. and Simon, B. (1979). Methods of modern mathematical physics, III. Scattering theory (Academic Press, New York), 462 p.

Richtmyer, R. (1978). Principles of advanced mathematical physics (Springer Verlag, New York), 422 p. 
Rytov, S., Kravtsov, Y. A., and Tatarskii, V. (1989). Principles of statistical radiophysics 3: Elements of random fields (Springer Verlag, Berlin), 239 p.

Sánchez-Sesma, F., Pérez-Ruiz, J., Campillo, M., and Luzón, F. (2006). "Elastodynamic 2D Green function retrieval from cross-correlation: Canonical inclusion problem", Geophys. Res. Lett. 33, L13305.

Sánchez-Sesma, F. J. and Campillo, M. (2006). "Retrieval of the Green's Function from Cross Correlation: The Canonical Elastic Problem”, Bull. Seism. Soc. Am. 96, 1182-1191.

Sato, H. (2009). "Retrieval of Green's function having coda from the cross-correlation function in a scattering medium illuminated by surrounding noise sources on the basis of the first order Born approximation", Geophys. J. Int. 179, 408-412.

Sato, H. (2010). "Retrieval of Green's function having coda waves from the cross-correlation function in a scattering medium illuminated by a randomly homogeneous distribution of noise sources on the basis of the first-order Born approximation", Geophys. J. Int. 180, 759-764.

Sens-Schönfelder, C. and Wegler, U. (2006). "Passive image interferometry and seasonal variations of seismic velocities at Merapi Volcano, Indonesia", Geophys. Res. Lett. 33, L21302.

Shapiro, B. (2011). "Green function correlations across physics subfields", Physics Today 64, 11.

Snieder, R. and Fleury, C. (2010). "Cancellation of spurious arrivals in Green's function retrieval of multiple scattered waves", J. Acoust. Soc. Am. 128, 1598-1605.

Snieder, R., Grêt, A., Douma, H., and Scales, J. (2002). "Coda wave interferometry for estimating nonlinear behavior in seismic velocity", Science 295, 2253.

Snieder, R. and Page, J. (2007). "Multiple scattering in evolving media", Physics Today 60, 49-55.

Snieder, R., Sánchez-Sesma, F. J., and Wapenaar, K. (2009). "Field Fluctuations, Imaging with Backscattered Waves, a Generalized Energy Theorem, and the Optical Theorem", SIAM J. Imaging Sciences 2, 763-776.

Snieder, R., van Wijk, K., Haney, M., and Calvert, R. (2008). "Cancellation of spurious arrivals in Green's function extraction and the generalized optical theorem", Phys. Rev. E 78, 036606.

Snieder, R., Wapenaar, K., and Wegler, U. (2007). "Unified Green's function retrieval by crosscorrelation; connection with energy principles", Phys. Rev. E 75, 36103.

van Rossum, M. and Nieuwenhuizen, T. (1999). "Multiple scattering of classical waves: microscopy, mesoscopy, and diffusion", Rev. Mod. Phys. 71, 313-371.

Varatharajulu, V. (1977). "Reciprocity relations and forward amplitude theorems for elastic waves", J. Math. Phys. 18, 537-543.

Wapenaar, K. (2004). "Retrieving the elastodynamic Green's function of an arbitrary inhomogeneous medium by cross correlation", Phys. Rev. Lett. 93, 254301.

Wapenaar, K. (2006). "Nonreciprocal Green's function retrieval by cross correlation", J. Acoust. Soc. Am. 120, EL7-EL13.

Wapenaar, K. and Fokkema, J. (2006). "Green's function representations for seismic interferometry", Geophysics 71, SI33-SI46.

Wapenaar, K., Slob, E., and Snieder, R. (2006). "Unified Green's function retrieval by cross correlation", Phys. Rev. Lett. 97, 234301. 
Weaver, R. and Lobkis, O. (2004). "Diffuse fields in open systems and the emergence of the Greens function (L)", J. Acoust. Soc. Am. 116, 2731-2734.

Wegler, U. and Sens-Schoenfelder, C. (2007). "Fault zone monitoring with passive image interferometry", Geophys. J. Int. 168, 1029-1033.

Yaglom, A. (2004). An introduction to the theory of stationary random functions (Dover Publications, New York), $235 \mathrm{p}$. 